\title{
A Waste Collection Problem with Service Type Option
}

\author{
Sina Gläser
}




\title{
A Waste Collection Problem with Service Type Option
}

\author{
Sina Gläser*
}

\begin{abstract}
Efficient solid waste management is one of the most relevant issues for urban communities. With regard to the service type of household waste collection, there are two approaches in practice: When collecting household waste via a door-to-door system, the collection vehicles drive down all the streets to empty the garbage cans on the curb. Using a bring system, waste is accumulated at central collection sites with a larger capacity. While residents do have to travel an additional distance to dispose of their waste at these central collection sites, a bring system can reduce the distance to be covered by collection vehicles because they no longer have to drive down all the streets. To combine the advantages of both systems, in this article the service type decision is introduced. The corresponding waste collection problem with service type option (WCPSTO) is modeled as a new variant of a periodic location routing problem and an adaptive large neighborhood search-based solution approach is proposed. This solution approach not only solves the newly presented problem, but also outperforms a solution algorithm for a related waste collection problem seen in the literature, which can be seen as a special case of the WCPSTO.
\end{abstract}

Keywords: Combinatorial optimization, Waste collection, Vehicle routing, ALNS

\section{Introduction}

Particularly in cities, waste collection faces a variety of safety, environmental and economic challenges. For example, maneuvering collection vehicles in crowded streets sometimes leads to fatal accidents and should therefore be avoided if possible. In addition, in rush hours, certain roads may be approached only during certain time windows. Waste collection itself increases the already high volume of traffic and thus also air polluting emissions in the cities. If there is only little space available for the collection of waste in densely built-up cities, the receptacles have to be emptied at a correspondingly higher frequency, which in turn increases traffic, emissions and waste collection costs. In the future, this problem will intensify: In 2018, the World Bank expected the daily per capita waste generation in high-income countries to increase by 19 percent by 2050 (Kaza et al. 2018), whereas in 2015 the OECD (2015) predicted that $85 \%$ of the world's population would live in cities by 2100 . This already results in referring to solid waste management as "one of modern society's most relevant issues" (Ghiani et al., 2014) and a "major concern for urban communities" (Gambella et al., 2019) in the literature.

*Bielefeld University, Department of Business Administration and Economics, sina.glaeser@uni-bielefeld.de 
In practice, there are different approaches to municipal solid waste collection. With regard to the type of service, two main approaches can be distinguished according to Pires et al. (2019, Chapter 3): A pickup system (PS), also called door-to-door collection or curbside collection, where households place their garbage cans on the street right next to their houses in accordance with a waste collection calendar. The second approach is a bring system (BS), where waste is accumulated at central collection sites with a larger capacity. Households have to cover a short distance to dispose of their waste at these central locations. Both systems offer advantages and disadvantages. In a PS, residents do not have to go any extra distance to dump their waste, and there is no need for opening central collection sites, which involves costs, e.g., for the development of the site. A BS, on the other hand, can reduce the distance to be covered by collection vehicles because they no longer have to drive along all streets nor stop at every house leaving the engine running. The idea in this article is to combine both service type approaches to benefit from the advantages of both systems. If, for example, it is not possible to set up a central collection site for a BS in a particular area because there is no suitable access for the collection vehicles or the road's bearing load is insufficient, the residents in that area can be served by a PS and do not necessarily have to cover extra distances for waste disposal. Such a mixed system can in fact be found in practice. In Germany, for example, households are typically served via a PS (European Environment Agency, 2016). This is the case in the city of Hamburg too. Space limitations meant that in some areas of the city not all the garbage cans could be put out on the curbside. Using extra garbage bags instead of more garbage cans initially seemed to be an effective, low-cost solution, but proved to be unhygienic and a traffic hazard - not to mention the blemished cityscape. Therefore, central collection sites have been set up and today, part of the households are served via a BS and in part via a PS (Stadtreinigung Hamburg, 2019; Bruck, 2020).

This article deals with the combinatorial problem that arises when introducing a mixed system. To do so, it has to be decided which households should be served via the PS and which via the BS. To serve households by means of the BS, it is necessary to open central collection sites (from a set of potential ones) to which households are assigned for their waste disposal. If households are served by the PS, they have to be provided with garbage cans. The greater the capacity of the garbage cans, the less collection visits are necessary. In context of a BS, the central collection sites must also be equipped with containers. Here, the emptying frequency additionally depends on the number of households assigned to these locations. The more households that dispose of their waste at a central collection site, the more frequently must this site be served for emptying. Given a required emptying frequency for both locations (households and central collection sites), determining on which days this should take place is also part of the problem. The aim is to minimize the weighted sum of the costs for (i) opening central collection sites and (ii) equipping them with containers, (iii) equipping households with garbage cans, (iv) the distance to be covered by collection vehicles and (v) the distance to be covered by residents to central collection sites for waste disposal. The last point represents a compensation payment, e.g., a waste fee reduction for the households involved, and should ensure that they accept the new mixed system and do not feel unfairly treated. Figure 1 illustrates the problem. The vehicles start and end their tours empty in the depot. Starting from here, they drive to the central collection sites, or the households. Once the capacity of a collection vehicle is reached, it is driven to a landfill, hereafter called intermediate facility (IF), to unload the collected waste and continue its tour. 
Figure 1: Waste collection with pickup and bring system.

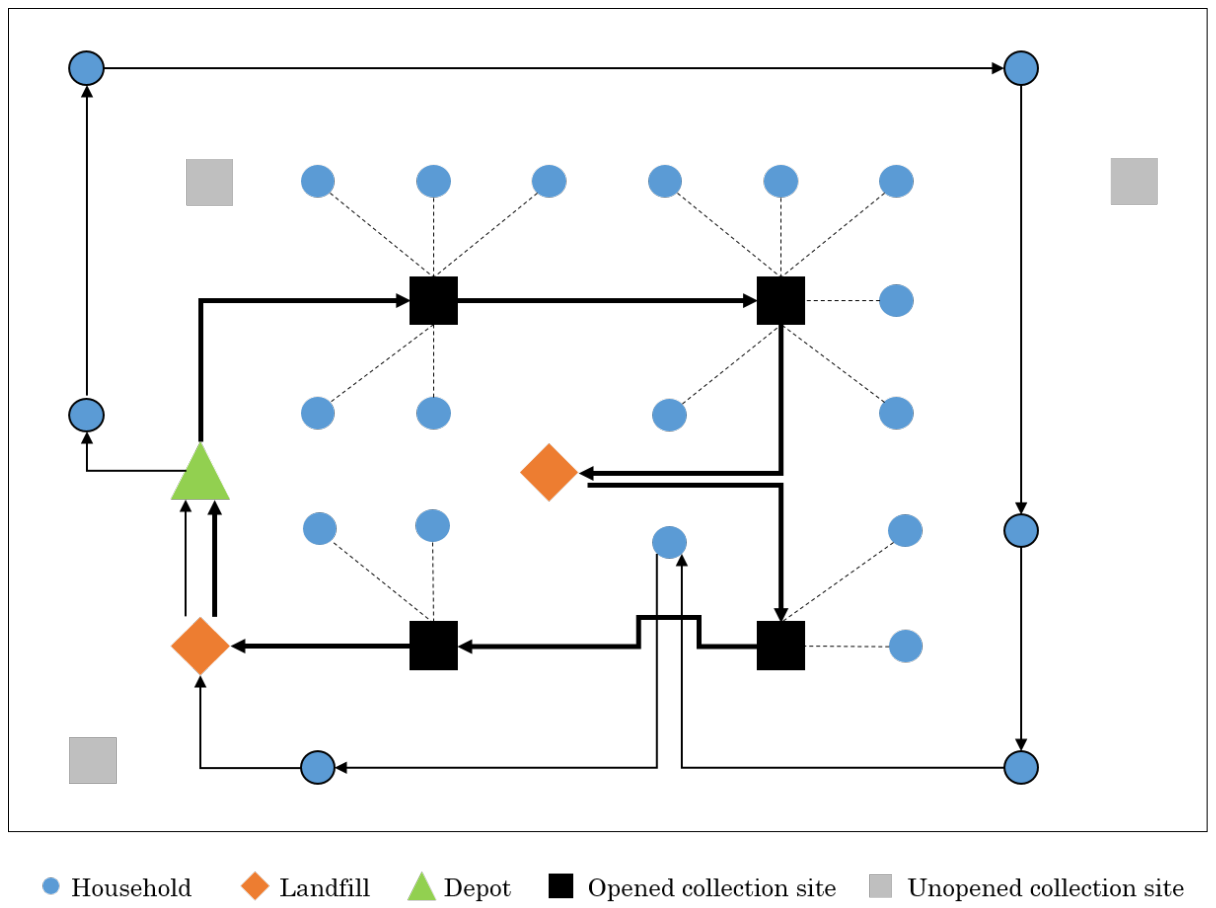

Note. Households are either assigned to a collection site or served directly at home by a (pickup) collection vehicle. For unloading, the vehicle is driven to a landfill. Costs arise from the black components: from the routes for the pickup collection vehicles (thin line), from the routes for the bring system vehicles (thick line), from the assignment of households to central collection sites (dashed line), by providing them with garbage cans and by the opening and equipping of central collection sites (black squares).

This article makes two main scientific contributions. First, an extended periodic location routing problem for a waste collection problem with service type option (WCPSTO) is modeled. In the context of the WCPSTO, new subtour elimination conditions for routing problems with intermediate facilities, which have not been used in the literature so far, are presented. Second, a solution approach which is based on an adaptive large neighborhood search (ALNS) algorithm is developed, which provides promising results also with respect to another problem from the waste collection context.

The remaining sections of the article are organized as follows. Section 2 gives an overview of the existing literature on related problems. Section 3 shows the mathematical model including the subtour elimination conditions already mentioned. The solution approach is presented in Section 4 . The results of the computational study can be found in Section 5 . Section 6 concludes the article with a summary of the most important findings and prospects on future research areas.

\section{Related work}

To the best of our knowledge, the WCPSTO, which is based on a location routing problem (LRP), is a new problem not yet covered in the literature. The LRP links the interdependent problems of vehicle routing and facility location. Salhi \& Rand (1989) showed already 30 years ago that separate investigation can lead to suboptimal solutions and an integrated approach to both problems is therefore useful. In the 
meantime, a huge number of different LRPs have been considered in the literature. A current overview can be found for example in the reviews by Prodhon \& Prins (2014), Drexl \& Schneider (2015) or Schneider \& Drexl (2017).

Closely related to the problem tackled here is the two-echelon location routing problem (2E-LRP) which comprises a location problem and a two-echelon vehicle routing problem (2E-VRP). An overview of two-echelon routing problems can be found in Cuda et al. (2015). In a 2E-VRP, the customers' demands are known, but are not served directly from the depot. They are first consolidated and brought to so-called satellite facilities. The version of this researched more than any other is the 2E-CVRP, where both the satellite facilities and the vehicles are capacitated (Cuda et al., 2015). In the WCPSTO, these facilities correspond to the capacitated central collection sites. The routes from the depot to the satellite facilities are called first-level routes. Customers are then served by second-level routes from the different satellite facilities (with smaller, eco-friendly vehicles for example). Split deliveries are allowed only on the first level. In the WCPSTO, split deliveries are not permitted on any level because all receptacles (containers and garbage cans) can be emptied completely only by the collection vehicles. A 2E-VRP variant without split deliveries is considered by Zhou et al.(2018) for a last mile parcel delivery problem. Apart from the existence of multiple depots, another special feature of their model is that on the second level not only customers, but also pickup facilities are served. From these, customers can pick up the parcels themselves when it suits them. Zhou et al. (2018) refer to their model as a multi-depot twoechelon vehicle routing problem with delivery options for the last mile distribution (MD-TEVRP-DO). A similar variant is used by Macrina et al. (2020) to model a crowd-shipping problem. On the firstlevel routes (starting from a depot), customers and so-called transshipment nodes are served by classical vehicles. The occasional drivers can then (starting from their personal location) pick up parcels from the depot and the transshipment nodes on the second-level routes in order to serve customers for a small compensation.

If, in addition to the first-level and second-level routes, the locations of the satellite facilities have also to be determined from a set of potential satellite facilities, it is called a 2E-LRP. If there is only one depot, it is referred to more precisely as a single depot 2E-LRP, otherwise as a multi-depot $2 \mathrm{E}$ LRP. A single-depot 2E-LRP, as it is relevant in the WCPSTO, is for example considered by Nguyen et al. (2012) in the context of city logistics. By placing so-called "intermediate depots" close to the city and serving customers using them instead of a single depot, they want to reduce inner-city traffic. They do not allow split deliveries, but customers may be served directly from the main depot. In the WCPSTO, this would correspond to a waste collection using a pickup system. To model this, Nguyen et al. (2012) place a fictitious satellite in the same place as the main depot. In contrast to the WCPSTO, on the second level there are no direct connections between the individual customers and the satellite facility, but several customers are served together within (second-level) tours. A 2E-LRP with direct connections between customers and satellite facilities is described by Zhou et al. (2017) inspired by a last mile parcel delivery problem. According to Zhou et al. (2017), customers can choose between a home delivery service or a pick-up service, where the customers collect their parcels from pickup stores (which correspond to satellite facilities) themselves. The probability that customers will choose a home delivery service increases with the distance to the nearest pickup store. These stores are then supplied accordingly on the first-level routes, and the customers who have chosen home delivery service are 
served with second-level routes. The problem is complicated by the fact that home delivery customers may not be at home, which leads to a failure, and a second delivery attempt must be made. A fact that indeed makes last mile parcel delivery more difficult (e.g., Akeb et al. 2018), but plays a minor role in the waste context. Another difference between the WCPSTO and the model of Zhou et al. (2017) is that customers do not necessarily have to dispose of their waste in the nearest container. Although the distance should be minimized, the assignment of customers to collection sites is part of the decision problem.

Taking into account periodicity is a typical extension in the waste collection context, which has not been considered in any of the work mentioned so far. For the first time the LRP was extended by periodicity in the work of Prodhon (2008), who proposed a local seach metaheuristic to solve the PLRP. Hemmelmayr et al. (2017) modeled a collaborative recycling problem as a PLRP; and more recently, Mamaghani \& Davari (2020) combine a PLRP with simultaneous pickup and delivery for the retrieval of packaging material. To solve the problem, they use a genetic algorithm. However, periodicity in the waste context is more often found in routing problems as a periodic vehicle routing problem (PVRP), typically in combination with the integration of intermediate facilities. These are used to unload the collected waste when the vehicle's capacity limit has been reached during a work shift so that it can continue its tour afterwards. Angelelli \& Speranza (2002b) introduced this problem as a periodic vehicle routing problem with intermediate facilities (PVRP-IF). Research on this in the waste context can be found for example in Angelelli \& Speranza (2002a), Coene et al. (2010) or Hemmelmayr et al. (2013). Later, Hemmelmayr et al. (2014) combined a PVRP-IF with the determination of the optimal service choice (SC) to a PVRP-IF-SC. In addition to determining the optimal emptying frequency for waste collection sites, their problem also involves determining on which days emptying should take place. In addition, Hemmelmayr et al. (2014) combined the problem with the question of the optimal capacity to be provided at the collection sites, which is why they call their problem the waste bin allocation and routing problem (WBARP). The location of the collection sites and the waste generation rates at them are known. Gläser \& Stücken (2021b) extended the model of Hemmelmayr et al. (2014) to a PLRPIF-SC by including the assignment of households to collection sites and the selection of these from a set of potential collection sites to model the introduction of an underground waste container system as a bring system. The daily volume of waste accumulated at the locations depends on the assignment of households to them. To ensure residents acceptance of the new waste collection system, the assignment of households to container locations is limited by the fact that residents can only be expected to travel a certain distance for waste disposal. However, Gläser \& Stücken (2021b) do not include a service option choice. All residents must dispose of their waste at central collection points.

\section{The waste collection problem with service type option}

The WCPSTO basically consists of two different subproblems connected by the service type option, which determines whether a household is served by the PS or the BS. In the following, the BS tours describe the tours serving the central collection sites of the BS, and the PS tours serve households via the PS. Households who are served by the PS are also referred to below as "customers". "Serving" in this case means the emptying of the receptacles in each case by a vehicle on a tour. Households 
not served by a PS are assigned to a central collection site (which in turn is served by a BS tour). If not specified more precisely, the term "site" below refers to both types of locations to be served, i.e. households and central collection sites. Since the determination of the central collection sites and the assignment of customers are also part of the BS subproblem, this part is the facility location and waste bin allocation and routing problem (FLWBARP) described by Gläser \& Stücken (2021a). Meanwhile, the decision about the PS tours and providing the customers (at home) with receptacles can be modeled by the WBARP introduced by Hemmelmayr et al. (2014), here referred to as the PS subproblem. For a more details about the related problems, the reader is therefore referred to Hemmelmayr et al. (2014) and Gläser \& Stücken (2021a). Below, the term "bin" is used specifically for receptacles at a customer's location and the term "container" refers to receptacles at central collection sites.

The problem at hand can be represented as a directed graph $G=(N, A)$, where $N$ is the set of nodes and $A$ is the set of arcs. The node set $N$ can be partitioned: The subset $N_{0}=\{0\}$ describes the depot where each vehicle starts and ends its route empty. Set $N_{C}$ represents the $n_{c}$ customers $N_{C}=\left\{1,2, \ldots, n_{c}\right\}$ which either have to be served by a PS tour or have to be assigned to a central collection site. $N_{P}=\left\{n_{c}+1, \ldots, n_{c}+n\right\}$ represents the $n$ potential central collection sites and $N_{I F}=\left\{n_{c}+n+1, \ldots, n_{c}+n+E\right\}$ the set of $E$ intermediate facilities. Each arc between a pair of nodes $i, j \in N$ is associated with a distance $d_{i j} \geq 0$, respecting the triangle inequalities. For simplicity, it is assumed that the travel time of a vehicle is equal to the distance $d_{i j}$. Not all potential central collection sites $i \in N_{P}$ and customers $j \in N_{C}$ need to be accessed by a collection vehicle $l$. The binary decision variables $h_{j}$ indicate whether a customer $j \in N_{C}$ is served by a PS tour $\left(h_{j}=1\right)$ or a BS tour $\left(h_{j}=0\right)$. If served by a BS tour, this customer is assigned to exactly one central collection site. The assigned location may not be further than $\bar{d}$ distance units from the customer's home. The central collection site in turn must be open and served by a vehicle. The binary decision variables $z_{i}$ determine whether a central collection site $i \in N_{P}$ is opened $\left(z_{i}=1\right)$ or not $\left(z_{i}=0\right)$ and the binary decision variables $\theta_{j i}$ indicate whether customer $j \in N_{C}$ is assigned to central collection site $i \in N_{P}\left(\theta_{j i}=1\right)$ or not $\left(\theta_{j i}=0\right)$. There is a cost of $C_{i}^{F}$ for opening a central collection site $i \in N_{P}$ and a cost of $\beta$ for each distance unit that an assigned customer $j \in N_{C}$ must cover to reach such a site for waste disposal.

Within the WCPSTO not only the routes of the vehicles are determined, but also on which days the tours should take place. Let $\mathrm{T}$ be the set of days $T=\{1, \ldots, 6\}$. The binary variable $f_{\text {ir }}$ indicates whether site $i \in N_{C} \cup N_{P}$ is assigned the frequency $r\left(f_{i r}=1\right)$ or not $\left(f_{i r}=0\right)$. According to Hemmelmayr et al. (2014) and Gläser \& Stücken (2021b) the possible visit combinations of the sites to be served are limited to symmetrical ones. This leads to twelve different visit combinations (VCs), which are listed in Table 1

It is assumed that each customer produces $w$ units of waste every day. The lower the frequency $r$, the greater the number of days $a_{r}$ on which there is no waste collection, the greater the amount of waste $q_{i}$ at a site $i\left\{\left\{i \in N_{P}: z_{i}=1\right\} \cup\left\{i \in N_{C}: h_{i}=1\right\}\right\}$ for which containers and bins, as the case may be, with sufficient capacity must be available to collect the waste until it is picked up the next time. At the central collection sites $i \in N_{P}$, the amount of waste $q_{i}$ depends not only on the selected frequency $r$ but also on the number of customers assigned to this central collection site.

Two different vehicle types are required for the two different levels of tours. Set $L_{B S}$ represents a total of $l_{B S}$ vehicles of type BS for the BS tours $L_{B S}=\left\{1, \ldots, l_{B S}\right\}$ and $L_{P S}$ represents $l_{P S}$ vehicles of type PS for the PS tours $L_{P S}=\left\{1, \ldots, l_{P S}\right\}$. Together, these sets form the set of all available vehicles 
Table 1: Overview of possible frequencies and the related visit combinations.

\begin{tabular}{|c|c|c|c|c|c|c|c|c|}
\hline \multirow[t]{2}{*}{ Frequency $r$} & \multirow{2}{*}{$\begin{array}{c}\text { Max. days between } \\
\text { two consecutive visits }\left(a_{r}\right)\end{array}$} & \multicolumn{6}{|c|}{ Day $t$} & \multirow{2}{*}{$\begin{array}{c}\text { Visit } \\
\text { combination } c\end{array}$} \\
\hline & & 1 & 2 & 3 & 4 & 5 & 6 & \\
\hline 1 & 6 & & & & & & & $\begin{array}{l}1 \\
2 \\
3 \\
4 \\
5 \\
6\end{array}$ \\
\hline 2 & 3 & & & & & & & $\begin{array}{l}7 \\
8 \\
9\end{array}$ \\
\hline 3 & 2 & & & & & & & $\begin{array}{l}10 \\
11\end{array}$ \\
\hline 6 & 1 & & & & & 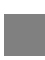 & & 12 \\
\hline
\end{tabular}

Note. The grey boxes represent the days on which a waste collection takes place.

$L=\left\{1, \ldots, l_{B S}, l_{B S}+1, \ldots, l_{B S}+l_{P S}\right\}$. Associated with each vehicle $l$ is a capacity $V_{l}$ to collect waste. If the capacity limit is reached, an IF must be approached for unloading. Afterwards the tour can be continued. The parts of a tour between the depot and an IF or between two IFs (which can be the same) are referred to as a trip. A tour can consist of several trips. There is a time limit $D$ that no tour may exceed. Not only the travel time has to be taken into account for this, but also the service time $s_{i}$ required for emptying the receptacles at a site $i \in N_{C} \cup N_{P}$. The variables $x_{i j l t}$ indicate whether the vehicle $l$ visits node $j$ on day $t$ immediately after node $i(i, j \in N, i \neq j)$. For each unit of distance traveled by a vehicle $l$, a cost of $\alpha_{l}$ is incurred. The load of the vehicle is described by the variable $v_{i j l t}$.

Different types of receptacles are available for waste collection at the sites, which are represented by the sets $B_{1}=\left\{1, \ldots, b_{1}\right\}$ and $B_{2}=\left\{1, \ldots, b_{2}\right\}$. Receptacles of the first type can be emptied only by a vehicle $l \in L_{1}$ and are therefore suitable only for the sites $i \in N_{P}$, so these are more precisely called containers. Receptacles of the second type can be emptied only by a vehicle $l \in L_{2}$, and are therefore suitable only for the sites $i \in N_{C}$ (bins). Associated with each receptacle of type $b \in B_{1} \cup B_{2}$ are purchase costs of $C_{b}^{F}$, a volume for waste collection $Q_{b}$, and a space requirement $u_{b}$. It should be noted that at each site $i$ only a certain amount of space $U_{i}$ is available for positioning the total number of $w_{i b}$ receptacles of type $b$ at site $i$. Thus, certain (low) collection frequencies may not be possible for a site because there is not enough space available to accommodate the waste accumulation.

As mentioned in Section 1, the aim is to minimize a weighted combination of costs for opening central collection sites, equipping them with containers and the customers with bins, routing by collection vehicles and covering the distance between customers' locations and central collection sites to dispose of waste in case of a BS.

The WCPSTO can be formulated as follows: 
Table 2: Indices used.

\begin{tabular}{ll}
\hline$b$ & Receptacle (container or bin) type index \\
$c$ & Visit combination index \\
$i, j, h, k$ & Indices for sites; where $i=j=h=k=0$ for the depot \\
$l$ & Vehicle index \\
$r$ & Number of visits (frequency) \\
$t$ & Day index \\
\hline
\end{tabular}

Table 3: Sets used.

\begin{tabular}{ll}
\hline$B_{1}$ & Set of container types, which can be served with a vehicle $l \in L_{B S}, B_{1}=\left\{1, \ldots, b_{1}\right\}$ \\
$B_{2}$ & Set of bin types, which can be served with a vehicle $l \in L_{P S}, B_{2}=\left\{1, \ldots, b_{2}\right\}$ \\
$C_{i}$ & Set of all possible visit combinations for site $i \in N_{P} \cup N_{C}, C_{i}=\{1, \ldots, 12\}$ \\
$L$ & Set of vehicles, $\left\{L_{B S} \cup L_{P S}\right\}$ \\
$L_{B S}$ & Set of vehicles of type $L_{B S}=\left\{1, \ldots, l_{B S}\right\}$ \\
$L_{P S}$ & Set of vehicles of type $L_{P S}=\left\{1, \ldots, l_{P S}\right\}$ \\
$N$ & Set of all nodes, $N=N_{0} \cup N_{C} \cup N_{P} \cup N_{I F}$ \\
$N_{0}$ & Set of depots, $N_{0}=\{0\}$ \\
$N_{C}$ & Set of customers, $N_{C}=\left\{1,2, \ldots, n_{c}\right\}$ \\
$N_{P}$ & Set of potential sites, $N_{P}=\left\{n_{c}+1, \ldots, n_{c}+n\right\}$ \\
$N_{I F}$ & Set of intermediate facilities, $N_{I F}=\left\{n_{c}+n+1, \ldots, n_{c}+n+E\right\}$ \\
$N_{1}$ & Set of nodes consists of $\left\{N_{C} \cup N_{P}\right\}$ \\
$N_{2}$ & Set of nodes consists of $\left\{N_{C} \cup N_{P} \cup N_{I F}\right\}$ \\
$N_{B S}$ & Set of nodes consists of $\left\{N_{P} \cup N_{0} \cup N_{I F}\right\}$ \\
$N_{P S}$ & Set of nodes consists of $\left\{N_{C} \cup N_{0} \cup N_{I F}\right\}$ \\
$N$ & Set of nodes consists of $\left\{N_{C} \cup N_{P} \cup N_{0} \cup N_{I F}\right\}$ \\
$R$ & Set of number of visits, $R=\{1,2,3,6\}$ \\
$T$ & Set of days, $T=\{1, \ldots, 6\}$ \\
\hline
\end{tabular}

$$
\min \sum_{\substack{i \in N \\ i \neq j}} \sum_{j \in N} \sum_{\substack{j \neq L \\ j \neq i}} \sum_{t \in T} \alpha_{l} d_{i j} x_{i j l t}+\sum_{b \in B_{1} \cup B_{2}} \sum_{i \in N_{1}} C_{b}^{P} w_{i b}+\sum_{i \in N_{P}} C^{F} z_{i}+\beta \sum_{i \in N_{C}} \sum_{j \in N_{P}} d_{i j} \theta_{i j}
$$

subject to

$$
\begin{array}{lr}
\sum_{i \in N_{P}} \eta_{i j}+h_{j}=1 & \forall j \in N_{C} \\
\eta_{i j}-\theta_{i j} \leq 0 & \forall i \in N_{P}, j \in N_{C} \\
\eta_{i j}-z_{j} \leq 0 & \forall i \in N_{P}, j \in N_{C} \\
z_{j}+\theta_{i j}-\eta_{i j} \leq 1 & \forall i \in N_{P}, j \in N_{C} \\
q_{i}=h_{i} w \sum_{r=1}^{R} a_{r} f_{i r} & \forall i \in N_{C} \\
\sum_{j \in N_{P}} d_{i j} \theta_{i j} \leq \bar{d} & \forall i \in N_{C} \\
q_{i}=\sum_{j \in N_{C}} \theta_{j i} w \sum_{r \in R} a_{r} f_{i r} & \forall i \in N_{P}
\end{array}
$$


Table 4: Parameters used.

\begin{tabular}{ll}
\hline$a_{c t}$ & Indicator whether day $t$ is contained in visit combination $c$ \\
$a_{r}$ & Maximum number of days between two consecutive visits depending on the total \\
& number of visits $r$ in the planning horizon \\
$\alpha_{l}$ & Cost factor for one distance unit traveled with vehicle $l$ \\
$b_{1}$ & Number of container types which can be unloaded by a vehicle $l \in L_{B S}$ \\
$b_{2}$ & Number of bin types which can be unloaded by a vehicle $l \in L_{P S}$ \\
$\beta$ & Cost factor for one distance unit traveled by a customer \\
$C_{b}^{P}$ & Purchase cost for a receptacle of type $b$ \\
$C_{i}^{F}$ & Cost for opening site $i \in N_{P}$ (opening cost rate) \\
$\bar{d}$ & Maximal distance a customer would accept to a central collection site (distance \\
& limit) \\
$d_{i j}$ & Distance/Travel time from site $i$ to $j$ \\
$D$ & Maximum tour length \\
$E$ & Number of intermediate facilities \\
$l_{B S}$ & Number of vehicles of type BS \\
$l_{P S}$ & Number of vehicles of type PS \\
$\lambda_{c r}$ & Indicator stating whether combination $c$ belongs to $r$ \\
$n_{c}$ & Number of customers \\
$n$ & Number of potential central collection sites \\
$Q_{b}$ & Volume capacity of a receptacle of type $b$ \\
$s_{i}$ & Service duration at site $i \in N_{1}$ \\
$u_{b}$ & Space needed by a receptacle of type $b$ \\
$U_{i}$ & Maximum of total available space for receptacles at site $i$ \\
$V_{l}$ & Volume capacity of vehicle $l \in L$ \\
$w$ & Amount of waste produced per day by a customer (waste generation rate) \\
\hline
\end{tabular}

$$
\begin{aligned}
& \sum_{b \in B_{1}} Q_{b} w_{i b}-q_{i} \geq 0 \\
& \forall i \in N_{P} \\
& \sum_{b \in B_{1}} w_{i b} u_{b} \leq U_{i} \\
& \forall i \in N_{P} \\
& \sum_{b \in B_{2}} Q_{b} w_{i b}-q_{i} \geq 0 \\
& \forall i \in N_{C} \\
& \sum_{b \in B_{2}} w_{i b} u_{b} \leq U_{i} \\
& \forall i \in N_{C} \\
& \sum_{c \in C_{i}} y_{i c}=z_{i} \\
& \forall i \in N_{P} \\
& \sum_{c \in C_{i}} y_{i c}=h_{i} \\
& \forall i \in N_{C} \\
& \sum_{c \in C_{i}} y_{i c} \lambda_{c r}=f_{i r} \\
& \forall i \in N_{1} ; r \in R \\
& \sum_{\substack{j \in N_{B S} \\
j \neq i}} \sum_{l \in L_{1}} x_{i j l t}-\sum_{c \in C_{i}} a_{c t} y_{i c}=0 \\
& \forall i \in N_{D} ; t \in T \\
& \sum_{\substack{j \in N_{P S} \\
j \neq i}} \sum_{l \in L_{2}} x_{i j l t}-\sum_{c \in C_{i}} a_{c t} y_{i c}=0 \\
& \forall i \in N_{C} ; t \in T
\end{aligned}
$$


Table 5: Decision variables used.

\begin{tabular}{|c|c|c|c|}
\hline$\delta_{i l t}$ & \multicolumn{2}{|l|}{ Integer variable } & \\
\hline$\eta_{i j}$ & \multicolumn{3}{|l|}{ Binary variable } \\
\hline$f_{i r}$ & \multicolumn{3}{|c|}{ Binary variable indicating whether $r$ is used for site $i \in N_{1}$ (1) or not (0) } \\
\hline$h_{i}$ & \multicolumn{3}{|c|}{ Binary variable indicating whether customer $i \in N_{C}$ is served via PS (1) or not (0) } \\
\hline$q_{i}$ & \multicolumn{3}{|c|}{ Amount of waste to be collected at site $i \in N_{1}$ at each visit } \\
\hline$\theta_{j i}$ & \multicolumn{3}{|c|}{$\begin{array}{l}\text { Binary variable indicating whether customer } j \in N_{C} \text { is assigned to central collection } \\
\text { site } i \in N_{P} \text { (1) or not (0) }\end{array}$} \\
\hline$v_{i j l t}$ & \multicolumn{3}{|c|}{ Real variable indicating the load of vehicle $l$ on arc $i j$ on day $t$} \\
\hline$w_{i b}$ & \multicolumn{3}{|c|}{ Number of receptacles of type $b$ allocated at site $i$} \\
\hline$x_{i j l t}$ & \multicolumn{3}{|c|}{$\begin{array}{l}\text { Binary variable indicating whether vehicle } l \text { visits site } j \text { immediately after site } i \text { on } \\
\text { day } t(1) \text { or not (0) }\end{array}$} \\
\hline$y_{i c}$ & \multicolumn{3}{|c|}{$\begin{array}{l}\text { Binary variable, indicating whether visit combination } c \in C_{i} \text { is assigned to site } i \in \\
N_{1}\end{array}$} \\
\hline$z_{i}$ & \multicolumn{3}{|c|}{$\begin{array}{l}\text { Binary variable, indicating whether central collection site } i \in N_{P} \text { is opened (1) or } \\
\text { not }(0)\end{array}$} \\
\hline & $\sum_{\substack{i \in N \\
i \neq h}} x_{i h l t}-\sum_{\substack{j \in N \\
j \neq h}} x_{h j l t}=0$ & $\forall h \in N ; l \in L ; t \in T$ & $(17)$ \\
\hline & $\sum_{j \in N_{2}} x_{0 j l t} \leq 1$ & $\forall l \in L ; t \in T$ & $(18)$ \\
\hline & $x_{i j l t} \leq \sum_{h \in N_{2}} x_{0 h l t}$ & $\forall i \in N_{2} ; j \in N ; l \in L ; t \in T$ & (19) \\
\hline & $\sum_{i \in N} \sum_{\substack{j \in N \\
j \neq i}}\left(d_{i j}+s_{i}\right) x_{i j l t} \leq D$ & $\forall l \in L ; t \in T$ & $(20)$ \\
\hline & $x_{i 0 l t}=0$ & $\forall i \in N_{1} ; l \in L ; t \in T$ & $(21)$ \\
\hline & $v_{i j l t}=0$ & $\forall i \in N_{I F} ; j \in N ; l \in L ; t \in T$ & $(22)$ \\
\hline & $v_{0 j l t}=0$ & $\forall j \in N ; l \in L ; t \in T$ & (23) \\
\hline & $\sum_{i \in N} v_{i j l t}+q_{j} \sum_{\substack{i \in N \\
i \neq j}} x_{i j l t}=\sum_{i \in N} v_{j i l t}$ & $\forall j \in N_{1} ; l \in L ; t \in T$ & (24) \\
\hline & $v_{i j l t} \leq V_{l} x_{i j l t}$ & $\forall i, j \in N ; l \in L ; t \in T$ & $(25)$ \\
\hline & $\eta_{i j} \in\{0,1\}$ & $\forall i \in N_{C} ; j \in N_{P}$ & (26) \\
\hline & $f_{i r} \in\{0,1\}$ & $\forall i \in N_{1} ; r \in R$ & (27) \\
\hline & $h_{i} \in\{0,1\}$ & $\forall i \in N_{C}$ & (28) \\
\hline & $q_{i} \geq 0$ & $\forall i \in N_{I}$ & (29) \\
\hline & $\theta_{m i} \in\{0,1\}$ & $\forall i \in N_{P} ; m \in N_{C}$ & (30) \\
\hline & $v_{i j l t} \geq 0$ & $\forall i, j \in N ; l \in L ; t \in T$ & (31) \\
\hline & $w_{i b} \geq 0$ and integer & $\forall i \in N_{1} ; b \in B$ & $(32)$ \\
\hline & $x_{i j l t} \in\{0,1\}$ & $\forall i, j \in N ; l \in L ; t \in T$ & (33) \\
\hline & $y_{i c} \in\{0,1\}$ & $\forall i \in N_{1} ; c \in C_{i}$ & (34) \\
\hline
\end{tabular}




$$
z_{i} \in\{0,1\}
$$

$$
\forall i \in N_{P}
$$

Constraints (1)-(4) ensure that each customer $j \in N_{C}$ is served either via a PS tour, or that he or she is assigned to exactly one open central collection site. If a customer is assigned to an open central collection site, constraints (6) ensure that the distance limit is complied with when assigning the customer to a site. The amount of waste $q_{i}$ accumulating at a customer's place $\left(i \in N_{C}\right.$ ) between two consecutive visits of the waste collection vehicle depends on the waste generation rate $w$ and the waste collection frequency $r$ and is calculated according to constraints (5). According to constraints (7), at the central collection sites $i \in N_{P}$, the amount of waste $q_{i}$ additionally depends on the number of customers assigned to this site. Note, that for clarity, some constraints are shown in a non-linear form here. The model in a linear form can be found in the appendix. Constraints $(8)$ ensure that the capacity requirement by assigning customers to central collection sites does not exceed the existing capacity. Constraints 9 ) ensure that at the same time the available space $U_{i}$ is not exceeded at the sites $i \in N_{P}$. Constraints (10) and (11) ensure this for the sites $i \in N_{C}$. Constraints (12) and (13) ensure that each open central collection site and each customer who is served by a PS tour are assigned to exactly one VC $c$ that is permitted for the site. The binary variables $y_{i c}$ take the value 1 , if $\mathrm{VC} c$ is assigned to site $i$ (otherwise $y_{i c}=0$ ). The binary parameters $\lambda_{c r}$ indicate whether a selected VC $c$ corresponds to a selected frequency $r\left(\lambda_{c r}=1\right)$ or not $\left(\lambda_{c r}=0\right)$. Constraints (14) ensure that the selected frequency $r$ also matches the VC $c$ selected for the site. Constraints (15) and (16) guarantee that a site is visited only on the days of the assigned VC with an appropriate vehicle type for the respective level. Constraints (17) ensure that the vehicle leaves the site on these days. Constraints (18) and (19) ensure that each vehicle can leave the depot only once a day if at least one site has to be visited. Each vehicle can therefore serve only one route per day. Constraints 20] ensure that the maximum length $D$ of a tour is observed. Constraints 21) ensure that before returning to the depot at the end of a tour, each vehicle first drives to an IF to unload the waste and thus arrives empty at the depot. Constraints (22) and 23) ensure that the vehicles are fully unloaded when leaving an intermediate facility or the depot. The so-called flow constraints 24 specify that the outbound flow of accumulated waste from a site equals the inbound flow of waste plus the waste picked up at the site itself. Furthermore, governed by constraints (25), the capacity $V_{l}$ of each vehicle is taken into account. Constraints 26) to 35 are non-negativity constraints and an indication of which variables are binary and integer.

Finally, subtour elimination constraints are needed. The flow constraints 24) are not sufficient to avoid subtours because the load of a vehicle is (according to constraints (22) ) zero after it has visited an IF. To avoid subtours in the context of IFs, this article therefore uses the standard Miller-Tucker-Zemlin variables $\delta_{i l t}$, which reflect a rank order in which nodes are visited. Let $\delta_{i l t}$ be the Miller-Tucker-Zemlin variables, then the standard Miller-Tucker-Zemlin constraints used here are:

$$
\begin{array}{lr}
\delta_{i l t} \geq 1 \text { and integer } & \forall i \in N_{1} ; l \in L ; t \in T \\
\delta_{i l t} \leq\left(n_{c}+n\right) & \forall i \in N_{1} ; l \in L ; t \in T \\
\delta_{i l t}-\delta_{j l t}+1 \leq\left(n_{c}+n\right)\left(1-x_{i j l t}\right) & \forall i, j \in N_{1} ; l \in L ; t \in T .
\end{array}
$$

Furthermore, in order to avoid subtours as in Figure $2 \mathrm{a}, \delta_{j l t}$ shall also be at least one unit greater than 
Figure 2: Subtour elimination

a)

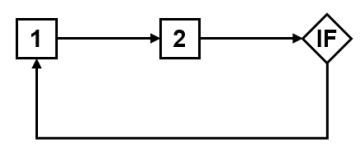

b)

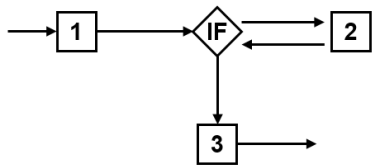

c)

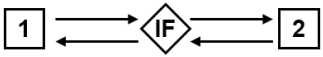

Note. The subtour eliminations presented here apply to both BS and PS tours.

$\delta_{i l t}$, if site $i$ and $j\left(i, j \in N_{1} ; i \neq j\right)$ are connected via an IF (and not directly). The constraints (38) are therefore also extended to

$$
\begin{gathered}
\delta_{i l t}-\delta_{j l t}+1 \leq\left(n_{c}+n\right)\left(1-x_{i j l t}-\sum_{h \in N_{I F}} \max \left(x_{i h l t}+x_{h j l t}-1 ; 0\right)\right) \\
\forall i, j \in N_{1} ; i \neq j ; l \in L ; t \in T .
\end{gathered}
$$

This eliminates a subtour as shown in Figure 2 a. The special case $i=j$ in constraints $(39)$ is excluded, to allow a connection as in Figure $2 \mathrm{~b}$. However, at the same time, this results in allowing a subtour as in Figure22. For the special case $i=j$ additional constraints are needed. Following the idea of the subtour elimination constraints by Dantzig et al. (1954), this is provided by the following constraints:

$$
\begin{gathered}
\delta_{i l t}-\delta_{j l t}+1 \leq\left(n_{c}+n\right) . \\
\left(1-x_{i j l t}-\sum_{h \in N_{I F}} \max \left(x_{i h l t}+x_{h j l t}-1 ; 0\right)+\sum_{h \in N_{I F}} \min \left(\max \left(x_{i h l t}-2+\sum_{k \in N_{P} \cup N_{0}} x_{h k l t} ; 0\right) ; x_{i h l t}\right)\right) \\
\forall i, j \in N_{P} ; i=j ; l \in L_{P S} ; t \in T \\
\delta_{i l t}-\delta_{i l t}+1 \leq\left(n_{c}+n\right) . \\
\left(1-x_{i j l g t}-\sum_{h \in N_{I F}} \max \left(x_{i h l t}+x_{h j l t}-1 ; 0\right)+\sum_{h \in N_{I F}} \min \left(\max \left(x_{i h l t}-2+\sum_{k \in N_{C} \cup N_{0}} x_{h k l t} ; 0\right) ; x_{i h l t}\right)\right) \\
\forall i, j \in N_{C} ; i=j ; l \in L_{B S} ; t \in T .
\end{gathered}
$$




$$
x_{i j l t}=0 \quad \forall i, j \in N_{I F} ; l \in L ; t \in T
$$

If two nodes $i$ and $j\left(i=j ; i, j \in N_{P}\right.$, respectively $\left.i, j \in N_{C}\right)$ are connected via an IF $h$, the IF $h$ must still be connected to at least one node $k\left(k \neq i ; k \in N_{P} \cup N_{0}\right.$, or $\left.k \in N_{C} \cup N_{0}\right)$. Finally, constraints 42, ensure that two IFs are never approached directly one after the other. In summary, the constraints of the WCPSTO are constraints (1) to (42). As already mentioned, the model in a linear form can be found in the appendix. To the best of our knowledge, this type of subtour elimination has not yet been used for PVRPs with intermediate facilities. They are also particularly suitable for PVRPs with IFs and time windows, because $\delta_{i l t}$ can directly be replaced by a site's $\left(i \in N_{C} \cup N_{P}\right)$ service time. For a current overview of VRPs with intermediate stops in general, see Schiffer et al. (2019).

\section{An adaptive large neighborhood search heuristic for the WCPSTO}

To solve the WCPSTO, an adaptive large neighborhood search heuristic (ALNS) is used. The ALNS was first introduced by Ropke \& Pisinger (2006) and has already been successfully used to solve (P)LRPs (see, e.g., Hemmelmayr et al. (2017); Schiffer \& Walther (2018)) as well as VRPs (see, e.g., Hemmelmayr et al.(2012); Azi et al.(2014); François et al.(2019)). The ALNS explores different neighborhoods. Switching between these does not follow a fixed scheme, but is adaptive, depending on the neighborhood operators' past performance. In this way the ALNS is able to adapt to various characteristics of the individual instances and it is possible to include neighborhood operators that are effective only in some cases (Pisinger \& Ropke, 2010).

\subsection{Iterations}

As a starting solution, the procedure of Gläser \& Stücken (2021a) is used, which means that first all customers are assigned to central collection sites, which in turn are opened. If a customer cannot be assigned to any central collection site due to the distance limit, this customer will (always) be served by the PS. Let $s$ be the incumbent solution, $s^{\prime \prime}$ a new solution, generated by applying a neighborhood operator and subsequent local search procedure(s), and $f(s)$ and $f\left(s^{\prime \prime}\right)$ the corresponding target function values. As with Hemmelmayr et al. (2014), and Gläser \& Stücken (2021a), not only solutions which yield an improvement in the target function value $\left(f\left(s^{\prime \prime}\right)<f(s)\right)$ are accepted. Based on a simulated annealing (SA) approach, also worse solutions are accepted with a certain probability $p\left(s, s^{\prime \prime}\right)$. The use of SA is quite common in combination with an ALNS (see, e.g., Ropke \& Pisinger (2006), Demir et al. (2012), Goeke \& Schneider (2015)). For a detailed description of the simulated annealing process and the acceptance decision the reader is referred to Hemmelmayr et al. (2014).

The neighborhood operators are selected by means of a roulette wheel procedure based on their weights, as described by Ropke \& Pisinger (2006). The better the operators $\kappa$ have performed in the past, the greater their weights $\omega_{\kappa}$ and thus the more likely are they to be selected. At the beginning of the procedure, all weights are equally distributed and all scores of the neighborhood operators are set to zero. An update of the weights takes place every $\tilde{t}$ iterations and thus divides the search phase into different segments. Let $\tilde{w}_{\kappa \Theta}$ be the weight of the operator $\kappa$ in the segment $\Theta$, then the weight of the 
operator $\kappa$ in the following segment $\Theta+1$ equals

$$
\tilde{w}_{\kappa \Theta+1}=\tilde{w}_{\kappa \Theta}(1-\tilde{r})+\tilde{r} \frac{\tilde{\pi}_{\kappa}}{\pi_{\kappa}} .
$$

Here, $\tilde{r}$ is the so-called reaction factor $(\tilde{r} \in(0,1)), \tilde{\pi}_{\kappa}$ is the score of operator $\kappa$ and $\pi_{\kappa}$ the number of times operator $\kappa$ was used in the corresponding segment. After updating the weights, $\pi_{\kappa}$ and $\tilde{\pi}_{\kappa}$ are reset to zero. If a neighborhood operator is able to find a new best solution, its score increases by $\Omega_{1}$, if the incumbent solution is improved, the score increases by $\Omega_{2}$ and if a new solution is worse than the incumbent, but accepted due to SA, the score increases by $\Omega_{3}$.

Algorithm 1 shows an overview of the ALNS process. The heuristic is terminated after an iterations limit.

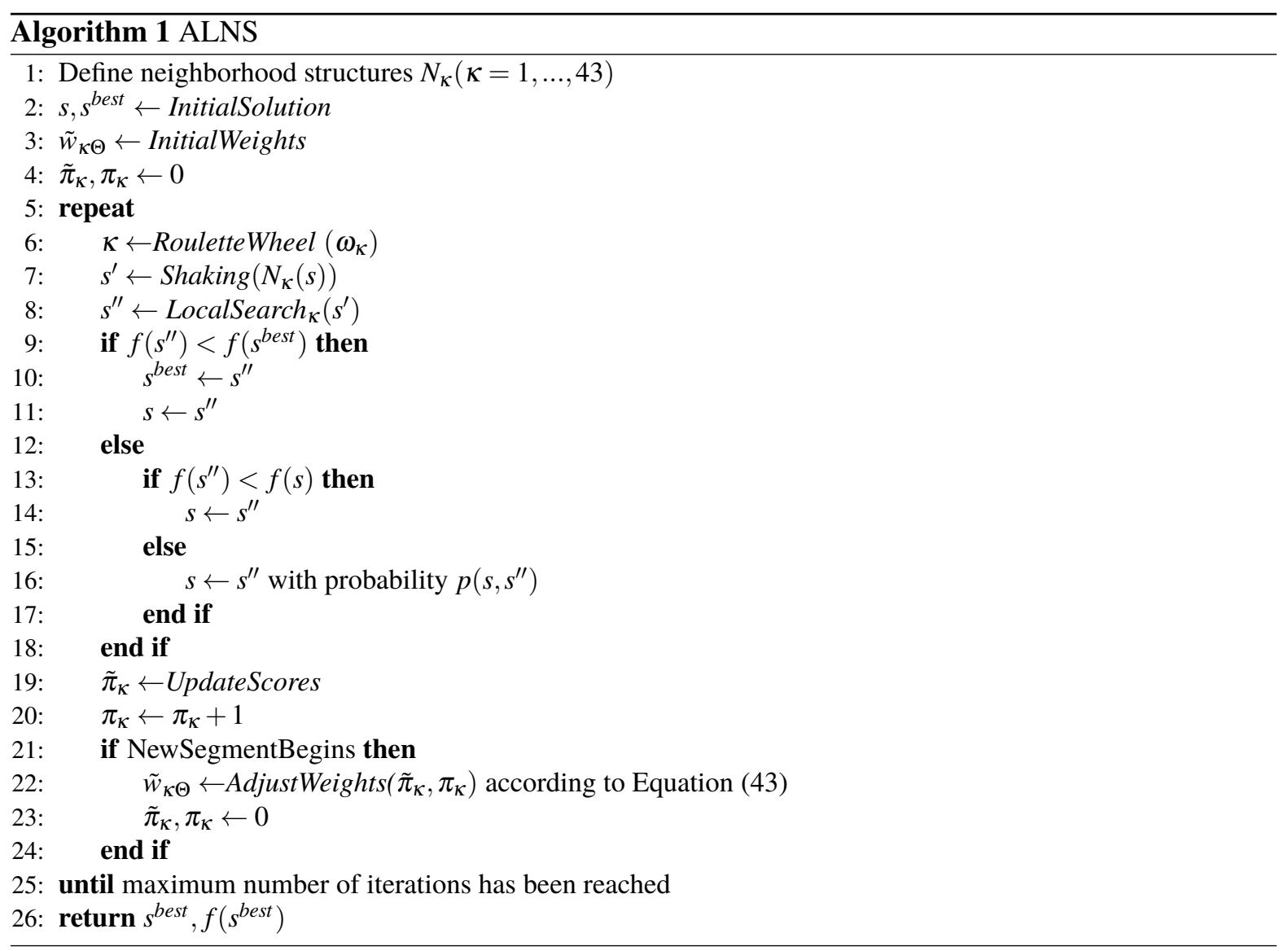

\subsection{Operators}

Note that, in the context of an ALNS, instead of explicit neighborhood operators, it is usually a question of so-called destroy and repair methods, which are selected independently of each other. In each iteration, the incumbent solution, or parts of it, are first destroyed by a destroy method, before being made feasible again by a repair method. The combination of different destroy and repair methods implicitly results in the neighborhoods. For the WCPSTO presented here, the 18 operators developed by Hemmelmayr et al. (2014) for the WBARP part and the 21 operators proposed by Gläser \& Stücken (2021a) 
for the FLWBARP part are adopted, and four more neighborhoods which link the two subproblems are added. Firmly connected to these 43 operators in total are associated local search procedures, which are performed after each application of a neighborhood operator to repair and improve the new solution. So basically, the standard components of an ALNS (destroy and repair methods) are used, but they cannot be freely combined. Pisinger \& Ropke (2010) call this a special case of the so-called coupled neighborhoods.

Table 6 shows an overview of all operators and the associated local search procedures used within the ALNS for the WCPSTO. The numbering serves only to identify the individual operators and represents no explicit order of operators. The four newly developed operators 40-43 and subsequently the local search procedures 1-3 are described in detail below. For the adopted operators 1-39, the reader is referred to the relevant authors. A detailed list of the individual operators can be found in the appendix in Table 21

Operator SWAP BS Assignment: Customers currently assigned to a central collection site $j$ are reassigned to another already opened central collection site $k \neq j$. The number of customers to be reassigned within the operator is determined by $\alpha^{B S}$, which determines how often a customer $i$ is chosen randomly from the set of all customers for reassignment. If customer $i$ is currently served by the BS, he or she is a candidate for reassignment. From the set of permissible central collection sites goverened by the distance limit for customer $i$, a new central collection site $(k \neq j)$ is chosen randomly for assignment. If no other central collection site is permissible for customer $i$ due to the distance limit, the current assignment is retained, and a new candidate is determined. Otherwise, customer $i$ will be assigned to site $k$. To make room for customer $i$, a randomly selected customer $m$, who is currently assigned to the central collection site $k$, has to be de-assigned from the site. In return, customer $m$ must now be reassigned to another collection site. To do so, it is first checked whether there is free capacity at the opened site that is closest to him/her (first-choice location) so that customer $m$ can dispose of his/her waste there. Otherwise, the second-choice location is checked, and so on. Note that the determining factor is not whether a collection site actually has sufficient capacity given the current container equipment and the current frequency of waste collection, but whether capacity would be available if the maximum container capacities and the highest frequency were to be used. Gläser \& Stücken (2021a) refer to this as the "maximum capacity of a site". If customer $m$ cannot be reassigned to an opened central collection site within the distance limit, the operator stops and the changes are reversed. If the operator was not stopped, LocalSearch2 (which will be explained in the further course of the section) is performed afterwards.

Operator SWAP PS $\rightarrow$ BS: A PS customer is randomly selected to potentially change from being a PS customer to being a BS customer. To do so, the PS customer must be assigned to an opened central collection site. There is a check which central collection site $j$ can be reached within the maximum distance limit and whose maximum capacity $Q_{j}^{\max }$ has not yet been reached. The first central collection site $j$ to be checked is determined randomly. If the first site $j \in N_{P}$ does not meet the requirements, the remaining opened central collection sites are checked in ascending order $\left(j+1, \ldots, n_{c}+n, n_{c}, \ldots, n_{c}+j-1\right)$. If the PS customer cannot be assigned to an opened 
Table 6: Operators and local search procedures for the WCPSTO

\begin{tabular}{lllll}
\hline$\kappa$ & Description & Local Search & Subproblem & \multicolumn{1}{l}{ Explanation } \\
\hline $1-3$ & Change combination & 1 & PS & Hemmelmayr et al. $(\overline{2014})$ \\
$4-15$ & Intertrip/-tour move/cross & 1 & PS & Hemmelmayr et al. $(\overline{2014})$ \\
$16-18$ & Change frequency & 1 & PS & Hemmelmayr et al. $(\overline{(2014})$ \\
$19-21$ & Change combination & 1 & BS & Hemmelmayr et al. \\
$22-33$ & Intertrip/-tour move/cross & 1 & BS & Hemmelmayr et al. \\
$34-36$ & Change frequency & 1 & BS & Hemmelmayr et al. $(\overline{2014})$ \\
$37-39$ & ADD/DROP/SWAP site & 2 & BS & Gläser \& Stücken $(2021)$ \\
40 & SWAP BS Assignment & 2 & BS & New \\
41 & SWAP PS $\rightarrow$ BS & $2 \& 3$ & BS \& PS & New \\
42 & SWAP BS $\rightarrow$ PS & $2 \& 3$ & BS \& PS & New \\
43 & DROP site - PS option & $2 \& 3$ & BS \& PS & New \\
\hline
\end{tabular}

Note. Operators 19-36 correspond to operators 1-18, so for these operators the reader is also referred to Hemmelmayr et al. (2014).

central collection site within the distance limit, the operator stops and the changes are reversed. If the operator was not stopped, LocalSearch2 and LocalSearch3 are performed afterwards.

Operator SWAP BS $\rightarrow$ PS: A BS customer is selected depending on the distance to his/her currently assigned central collection site using the roulette wheel method and becomes a PS customer. The greater the distance the customer currently has to travel to dispose of his/her waste, the more likely he or she is selected for being served by the PS. Subsequently, LocalSearch3 is applied.

Operator DROP site - PS option: Similar to the DROP operator of Gläser \& Stücken (2021a), this operator closes a central collection site, and the customers assigned to this now closed central collection site are reassigned among the remaining opened central collection sites. If not all customers can be reassigned according to the procedure described in Gläser \& Stücken (2021a), these customers are served by a PS tour. In the worst case, all customers that were assigned to the now-closed central collection site become PS customers. LocalSearch2 and LocalSearch3 are performed afterwards.

LocalSearch1: This local search is based on the local search procedure proposed by Hemmelmayr et al. (2014). After applying a neighborhood operator, the IFs for each modified tour (BS or PS) are removed and then reinserted. In the case of BS tours, this is done using dynamic programming (DP). In the case of PS tours, which can typically serve a larger number of customers until an IF has to be approached for emptying, the IFs are reinserted using greedy insertion. An IF is inserted into the tour right before the vehicle capacity would be exceeded. Such a greedy procedure is also used by Hemmelmayr et al. (2013) for large instances. Through both procedures, the solution is repaired to meet the capacity limits of the vehicles. Finally, the routes are improved using a 2-opt-method. Each time an operator's application changes the amount of waste $q_{j}$ that needs to be collected at a site $j$ until the next pickup, an optimal receptacle allocation is calculated using CPLEX.

LocalSearch2: This local search procedure is based on the local search procedure proposed by Gläser 
\& Stücken (2021a) and used after operators which have impact on the daily waste generation rate at central collection sites. The amount of waste accumulated daily at a central collection site changes if collection sites are opened or closed and customers are reassigned between central collection sites or become PS customers. As part of the LocalSearch2 procedure, in a first step, the assigned frequency is checked and, if necessary, repaired. If the central collection site was already open before and additional customers were assigned to this site by the operators $\kappa \in\{40,41,43\}$, the currently assigned frequency may no longer be feasible because more waste now accumulates daily at this site. In this case, a VC with the next higher feasible frequency is assigned to this central collection site, which contains the "old" VC. If, for example, VC 1 was assigned to the site before and a corresponding frequency of 1 is no longer sufficient, but frequency 2 would be sufficient, then VC 7 would be assigned to the central collection site. VC 7 (in contrast to the other VCs with frequency 2) already contains VC 1 and thus the day $t=1$. The central collection site must now be visited additionally only on day $t=4$. If a central collection site is newly opened by operator $\kappa \in\{37,39\}$, then this site is always assigned the highest feasible frequency to ensure that a feasible container allocation exists under any circumstances. In a second step, the integration into a BS tour of the corresponding additional days is done by Best Insertion. As an extension to LocalSearch2 by Gläser \& Stücken(2021a), this procedure is also used in the reverse case, when the amount of waste at a central collection site has decreased after applying the operators $\kappa \in$ $\{38,40,42,43\}$. If, for example, it suffices to visit a site once a week instead of twice a week, that central collection site is deleted from the tour schedule(s) accordingly. Central collection sites are also removed from the tour schedule if their daily waste generation rate equals zero. This can result from a reassignment of customers or closure of those sites. After LocalSearch2, LocalSearch1 is applied to improve the modified BS tours.

LocalSearch3: Similiar to LocalSearch2, LocalSearch3 is applied if the daily amount of waste at a PS customer site has changed. For a customer, who was previously served by the BS and has become a PS customer by the operators $\kappa \in\{42,43\}$, a VC is chosen randomly from the subset of all possible VCs with the lowest frequency. According to this $\mathrm{VC}$, the customer is assigned to the corresponding tour(s) using Best Insertion. If, on the other hand, a PS customer becomes a BS customer by the neighborhood operator $\kappa=41$, the amount of waste deposited at his/her sites becomes zero and the customer is deleted from the PS tours. In both cases, the PS tours have changed and LocalSearchl is applied afterwards to improve the modified PS tours.

\section{Computational Study}

Below, the calculations performed are explained and the results obtained are analyzed. First, the instances and parameter settings used are described in Section 5.1 before analyzing in Section 5.2 the efficiency of the developed ALNS which is implemented in Python. Computational results are presented and discussed in Section 5.3 All calculations were performed on an Intel Core with $3.0 \mathrm{GHz}$ CPU and 8 GB memory. The CPLEX solver (version 12.8) of the commercial software package AIMMS was used and called directly in Python within the framework of the ALNS. 


\subsection{Instances and parameter settings}

The instances used here are obtained by extending instances from the literature. Gläser \& Stïcken (2021a) provide instances for the FLWBARP, which serve as a basis for the WCPSTO considered here. In more detail, the Instances 01, 02 and 07 by Gläser \& Stücken (2021a) are used. For each of these instances, the first $n_{c}=500,1000$ and 1500 customers are used, which results in three instance sets (Set $\mathrm{a}$, Set $\mathrm{b}$, Set c) and a total of 9 instances. This enables us to analyze the influence of the number of customers to be served on the overall system in Section 5.3. An overview of the instance data used here can be found in Table 7 .

If customers are served by the PS, the distances between the individual customers, the IFs and the depot are calculated using the Euclidean distance measure. The capacity $V$ and the maximum tour length $D$ are assumed to be the same for both vehicle types (PS and BS). The service time $s_{i}$ for waste collection is set to 0.001 for all sites $i \in N_{C}$. The service times for all sites $i \in N_{P}$ are provided by Gläser \& Stücken (2021a). In addition to the number of customers $n_{c}$, number of IFs $E$ and potential central collection sites $n$ per instance, the table also shows two different maximum distance limits per instance. The distance limit on a high level $\bar{d}_{\text {high }}$ makes it possible for each customer to reach at least $50 \%$ of the potential central collection sites. If the distance limit is low $\left(\bar{d}_{\text {low }}\right)$, each customer can reach at least $10 \%$ of the potential central collection sites.

The container types for the central collection sites and the available space $U_{i}$ for all sites $i \in N_{P}$ in the bring system are taken from Gläser \& Stücken (2021a). Note that the data set of Gläser \& Stücken (2021a) is based on the assumption that 40 customers are assigned to one central collection site and that central collection sites are equipped with underground waste containers. For the PS, it is therefore assumed that the bin capacity and costs variants for the PS customers equal one fortieth of the corresponding BS figures for the underground waste containers. However, the space needed for bins amounts to $3 / 40$ ths of the space requirement for the underground waste containers because underground waste containers require on average only $1 / 3$ of the space required by ground-level containers (SULO Deutschland $\mathrm{GmbH}, 2021$ ). Table 8 shows an overview of all receptacle types used here and their characteristics.

The available space $U_{i}$ at a customer $i \in N_{C}$ is drawn randomly from the equally distributed inter-

Table 7: Overview of parameter values in the context of the WCPSTO

\begin{tabular}{cccccccccccc}
\hline Set & Instance & $n$ & $n_{c}$ & $\mathrm{E}$ & $\bar{d}_{\text {low }}$ & $\bar{d}_{\text {high }}$ & $V$ & $\mathrm{D}$ & $w$ & $l_{B S}$ & $l_{P S}$ \\
\hline \multirow{4}{*}{$\mathrm{a}$} & 01 & 48 & 500 & 4 & 50 & 105 & 150 & 600 & 0.34 & 2 & 2 \\
& 02 & 48 & 1,000 & 4 & 50 & 105 & 150 & 600 & 0.34 & 2 & 2 \\
& 03 & 48 & 1,500 & 4 & 50 & 105 & 150 & 600 & 0.34 & 2 & 2 \\
\hline \multirow{4}{*}{$\mathrm{b}$} & 04 & 96 & 500 & 4 & 50 & 132 & 200 & 1,150 & 0.32 & 2 & 2 \\
& 05 & 96 & 1,000 & 4 & 50 & 132 & 200 & 1,150 & 0.32 & 2 & 2 \\
& 06 & 96 & 1,500 & 4 & 50 & 132 & 200 & 1,150 & 0.32 & 2 & 2 \\
\hline \multirow{4}{*}{$\mathrm{c}$} & 07 & 72 & 500 & 6 & 50 & 112 & 175 & 950 & 0.33 & 2 & 2 \\
& 08 & 72 & 1,000 & 6 & 50 & 112 & 175 & 950 & 0.33 & 2 & 2 \\
& 09 & 72 & 1,500 & 6 & 50 & 112 & 175 & 950 & 0.33 & 2 & 2 \\
\hline
\end{tabular}

Note. Instances 01-03 are based on Instance 01 (I01) by Gläser \& Stücken (2021a), Instances 04-06 are based on I02, and Instances 07-09 are based on I07. Here, in comparison to the instances by Gläser \& Stücken (2021a), there are additionally two PS type vehicles. 
Table 8: Different receptacle types and their characteristics

\begin{tabular}{cccc}
\hline Bin type & Space requirement $u_{b}$ & Capacity $Q_{b}$ & Purchase cost $C_{b}^{P}$ \\
\hline$b_{B S}=1$ & 1 & 10 & 10 \\
$b_{B S}=2$ & 2 & 15 & 11 \\
$b_{B S}=3$ & 4 & 25 & 13 \\
\hline$b_{P S}=1$ & 0.075 & 0.250 & 0.250 \\
$b_{P S}=2$ & 0.150 & 0.375 & 0.275 \\
$b_{P S}=3$ & 0.300 & 0.625 & 0.325 \\
\hline
\end{tabular}

val $[0.15 ; 0.975]$. Thus, there is sufficient space available (at least 0.15 ) so that a volume of at least 0.375 can be provided at each customer site with a bin of PS type 2. This is sufficient to collect the waste volume accumulated in one day. In this case, however, a daily waste collection (which equals a visit frequency of $r=6$ ) is necessary. A space of 0.975 is in turn enough to provide sufficient capacity to collect the largest daily amount of waste $(w=0.34)$ for six days ( 2.04 in total), based on the choice of the most inexpensive bin equipment ( 3 bins of PS type 3 plus one bin of PS type 1). If the distance between a customer $i \in N_{C}$ and the depot is above average, meaning $\frac{\delta_{i 0}}{\sum_{m=1}^{N_{C}} \delta_{m i} / N_{C}} \geq 1$, the available space is drawn randomly from the interval $[0.675 ; 0.975]$. At these customer sites, there is always enough space for a waste collection with frequency $r=1$. This is based on the idea that there is more space in the outer cities than in the inner cities to place bins. However, the greater the space available at these customers' locations, the more inexpensively can the required capacity be provided.

The other parameter settings are also taken by Gläser \& Stücken (2021a), which results in each distance unit between a customer and his/her assigned central collection site being penalized by $\beta=0.0005$ (in the case of being served by the BS) and the cost factor for one distance unit traveled by a vehicle $\alpha_{l}$ is set to $1 \forall l \in L$. For opening a central collection site, costs in the amount of $C^{F}=5$ are incurred.

As part of the selection of operators, after every 1,000 iterations, the weights are adjusted according to the scores achieved. Inspired by Ropke \& Pisinger (2006), $\Omega_{1}$ is set to 33, $\Omega_{2}$ is set to 9 and $\Omega_{3}$ is set to 13. The reaction factor is set to $\Lambda=0.1$. As part of the SWAP BS Assignment operator, $\alpha^{B S}$ is drawn randomly from the uniformly distributed interval $[1 ; 20]$. This way, in total, a maximum of 40 customers can be reassigned using this operator. This number is based on the ratio between central collection sites and customers already presented.

\subsection{Algorithm performance analysis}

To be able to assess the solution quality based on optimal solutions, small instances are generated, which can be solved in reasonable time by the commercial solver CPLEX. For this purpose, only the first 5 (10) customers and the first 5 (10) central collection sites are taken from Instance 01 and only one vehicle is provided per vehicle type. The results obtained by CPLEX are compared with those obtained by the ALNS. Within the comparison, the distance limit $\bar{d}$ is set to 105 (high level) and the number of customers to be reassigned in the ADD site operator is set to $\gamma=1$, therefore allowing all 5 (10) customers to be reassigned if a new central collection site is opened.

Table 9 shows an overview of the parameter values and the results of the comparison. The values 
Table 9: Comparison of the results generated by CPLEX with those generated by the ALNS

\begin{tabular}{rlllllllllllr}
\hline & & \multicolumn{1}{c}{ CPLEX } & & & & & & \multicolumn{3}{c}{ ALNS } \\
$n$ & $n_{C}$ & $E$ & $l_{B S}$ & $l_{P S} V$ & LB & UB & Gap (\%) & Time & Time to UB & UB & Time to UB \\
\hline 5 & 5 & 4 & 1 & 1 & 1 & 916.51 & 916.51 & 0.00 & 980.31 & 978.09 & 916.51 & 1.97 \\
5 & 5 & 4 & 1 & 1 & 5 & 191.59 & 191.59 & 0.00 & 3.66 & 3.11 & 191.59 & 0.35 \\
5 & 5 & 4 & 1 & 1 & 10 & 132.77 & 132.77 & 0.00 & 2.17 & 1.39 & 132.77 & 0.12 \\
5 & 5 & 4 & 1 & 1 & 20 & 74.94 & 74.94 & 0.00 & 1.44 & 1.44 & 74.94 & 0.41 \\
\hline 10 & 10 & 4 & 1 & 1 & 1 & 270.63 & $1,224.91$ & 352.61 & - & $10,276.69$ & $1,220.16$ & 81.45 \\
10 & 10 & 4 & 1 & 1 & 5 & 220.25 & 220.25 & 0.00 & $15,756.78$ & 907.93 & 220.25 & 148.21 \\
10 & 10 & 4 & 1 & 1 & 10 & 132.05 & 132.05 & 0.00 & 159.03 & 26.70 & 132.05 & 5.521 \\
10 & 10 & 4 & 1 & 1 & 20 & 94.45 & 94.45 & 0.00 & 12.78 & 12.79 & 94.45 & 7.13 \\
\hline
\end{tabular}

Note. "UB" stands for upper bound, "LB" stands for lower bound, and the gap is the difference between the UB and LB. The column "Time" shows the solving time in seconds required by CPLEX to verify the optimum, while the columns "Time to UB" show the time in seconds required to find the UB by CPLEX and the ALNS respectively.

are given for one calculation using CPLEX and one calculation using the ALNS. By using CPLEX, the optimal solution for 7 out of 8 instances can be found and verified. However, these solutions are found (faster) by the ALNS too. For one instance, CPLEX failed to obtain an optimal solution because it ran out of memory. The solution found by the ALNS for this instance is within the bounds found by CPLEX. The results show that the ALNS developed is able to determine optimal (or promising) solutions for the small instances considered. An optimal solution for the instance with $n_{c}=10, n=10$ and $V=5$ is illustrated in Figure 3. This solution demonstrates that the adjustments to the subtour elimination constraints in Section 3 were necessary. Without the adjustments to the constraints, this solution would not be feasible because case b) of Figure 2 applies here.

To evaluate the performance of the algorithm developed for the larger instances presented in Section 5.1. the results of the ALNS are compared with the results that can be obtained using the VNS presented by Gläser \& Stücken (2021a) for the WBARP. Using the cost factors proposed by Gläser \& Stïcken (2021a) as described above $\left(C_{i}^{F}=5 \forall i \in N_{P}, \beta=0.0005, \alpha=1\right)$, all customers are served through the BS. So basically, the WCPSTO is reduced to the WBARP, and the results of the two heuristic solution approaches can be compared in terms of solution quality. According to Gläser \& Stücken (2021a), the maximum number of customers to be reassigned in the ADD site operator is set to $\gamma=4$ for both algorithms, which means that a maximum of one quarter of the customers can be reassigned. The initial temperature for the SA $T_{0}=7$ is also adopted for both algorithms and is reduced every 1,000 iterations in such a way that it is 0 in the last iteration.

Table 10 shows the results of the comparison, in which five runs were performed per instance and algorithm. Using the initial temperature of $T_{0}=7$, as proposed by Gläser \& Stïcken (2021a) for the ALNS, the ALNS outperforms the VNS in terms of the average target function value, but shows higher computation times for the high distance limit. On average, across all runs, the average target function values obtained are $11.51 \%$ (25.85\%) lower than the corresponding average target function values of the VNS using the low (high) distance limit. However, in the worst case (for Instance 04 using a low distance limit), the results obtained by the ALNS are on average $2.01 \%$ higher than the ones obtained by the VNS. The results in Table 10 show that using $T_{0}=20$ as the initial temperature (instead of $T_{0}=7$ ) for the ALNS results in outperforming the VNS on every instance while reducing the computation time 
Figure 3: Representation of the optimal solution for the small instance with $n_{c}=10, n=10$ and $V=5$.

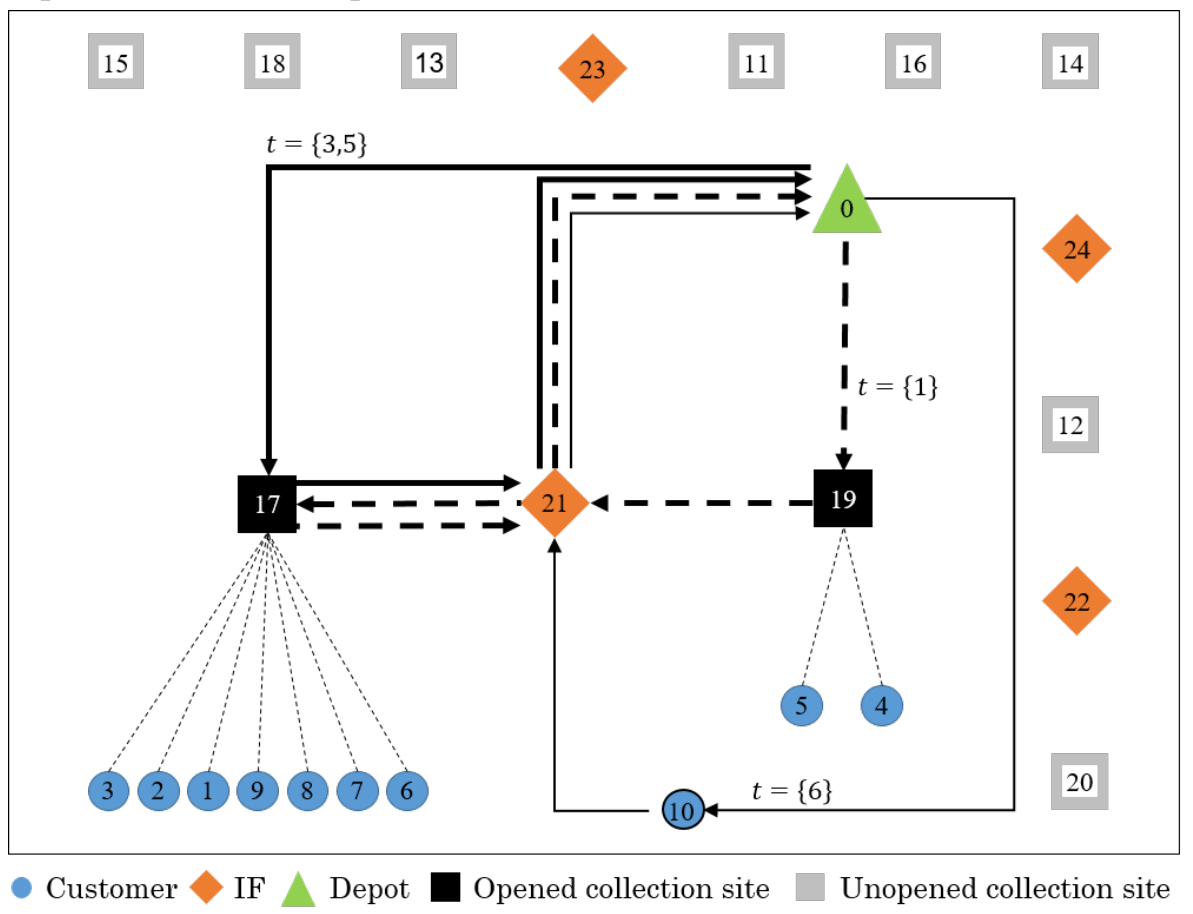

Note. Nine out of ten customer are assigned to central collection sites, customer $i=10$ is served by a $\mathrm{PS}$ vehicle on day $t=6$. However, every VC with frequency $r=1$ leads to the optimal target function value and represents an optimal solution. The central collection site $i=17$ needs to be approached three times per week.

in comparison to the $T_{0}=7$ setting. Therefore, $T_{0}$ is set to 20 in the following calculations. Tables 22 and 23 in the appendix give a detailed overview of the target function values of the individual instances. Note that the total number of iterations for a run for the VNS was set to $I_{V N S}=2 \cdot 10^{5}$, which, according to Gläser \& Stücken (2021a), is a good compromise between solution quality and run time for this algorithm. The total number of iterations of a run for the ALNS was set to $I_{A L N S}=10^{5}$. The results in Table 10 show that this represents a good tradeoff between solution quality and run time for the ALNS.

\subsection{Results}

Below, the results that could be obtained with the ALNS described above for the instances shown in Table 7 are interpreted.

\subsubsection{Sensitivity analysis regarding the opening cost rate.}

A sensitivity analysis regarding the opening cost rate for central collection sites is carried out. The results in the previous section showed that under the assumption of an opening cost rate for central collection sites of $C_{i}^{F}=5$, all customers are served by the BS. As shown below, this changes if higher opening cost rates for central collection sites are assumed. Two additional cost rates for opening a central collection site in the amount of $C_{i}^{F}=1,000$ and $C_{i}^{F}=10,000 \forall i \in N_{P}$ are considered below. For each cost rate, five runs of the ALNS were performed per instance and distance limit. Because no distinction is made 
Table 10: Comparison of the results generated by the VNS by Gläser \& Stücken (2021a) with those generated by the ALNS

\begin{tabular}{|c|c|c|c|c|c|c|c|}
\hline & & \multicolumn{3}{|c|}{$\bar{d}_{\text {low }}$} & \multicolumn{3}{|c|}{$\bar{d}_{\text {high }}$} \\
\hline & & $\begin{array}{l}\text { ALNS } \\
T_{0}=7\end{array}$ & $\begin{array}{c}\text { ALNS } \\
T_{0}=20\end{array}$ & VNS & $\begin{array}{l}\text { ALNS } \\
T_{0}=7\end{array}$ & $\begin{array}{c}\text { ALNS } \\
T_{0}=20\end{array}$ & VNS \\
\hline Avg. target function value & & $3,637.24$ & $3,539.41$ & $4,190.50$ & $1,507.52$ & $1,380.62$ & $2,060.42$ \\
\hline$\%$ deviation & worst & $+2.01 \%$ & $-5.12 \%$ & - & $0.00 \%$ & $-15.07 \%$ & - \\
\hline with respect & avg. & $-11.51 \%$ & $-14.96 \%$ & - & $-25.85 \%$ & $-33.64 \%$ & - \\
\hline to $\mathrm{VNS}$ & best & $-28.12 \%$ & $-28.38 \%$ & - & $-47.20 \%$ & $-51.59 \%$ & - \\
\hline Avg. time & & $5,458.01$ & $4,693.32$ & $5,682.72$ & $5,060.26$ & $4,444.28$ & $2,249.56$ \\
\hline
\end{tabular}

Note. The average values over 5 runs of the ALNS were determined per instance. These values are compared with the corresponding average values determined by the VNS. The worst and best average deviations of the ALNS values compared with the VNS are shown, as well as the average deviation value (avg.). "Avg. time" shows the average computing time in seconds over all instances and runs.

Table 11: Average share of PS customers, opened central collection sites and frequencies

\begin{tabular}{|c|c|c|c|c|c|c|c|c|c|}
\hline \multirow[b]{2}{*}{$\bar{d}$} & \multicolumn{3}{|c|}{$C^{F}=5$} & \multicolumn{3}{|c|}{$C^{F}=1,000$} & \multicolumn{3}{|c|}{$C^{F}=10,000$} \\
\hline & $\%$ PS & No. sites & $\varnothing r$ & $\%$ PS & No. sites & $\varnothing r$ & $\%$ PS & No. sites & $\varnothing r$ \\
\hline $\bar{d}_{\text {low }}$ & $0.00 \%$ & 14.64 & 4.82 & $16.82 \%$ & 8.31 & 5.86 & $19.36 \%$ & 8.07 & 5.89 \\
\hline $\bar{d}_{h i g h}$ & $0.00 \%$ & 8.64 & 5.93 & $0.56 \%$ & 6.64 & 5.99 & $9.97 \%$ & 5.80 & 6.00 \\
\hline
\end{tabular}

between the individual central collection sites $i$, the opening cost rates are presented from now on as $C^{F}$ (without index). The results are shown in Table 11, the share of customers served by the PS (\% PS), how many central collection sites are opened on average (No. sites) and with which frequency they are visited on average for waste collection $(\varnothing r)$ at each cost rate. A distinction is made between the two distance limits $\bar{d}_{\text {low }}$ and $\bar{d}_{\text {high }}$. In the case of an opening cost rate of 5, no customers are served by means of the PS (\% PS equals zero), regardless the distance limit. As the opening cost rate increases, there is a switch from a pure BS to a mixed system: On average, fewer central collection sites are opened which are visited with a higher frequency, and a greater share of customers are served by the PS. The results show that the share of customers served by the PS depends not only on the opening cost rate, but also to a great extent on the distance limit. The influence of the distance limit even seems to exceed the influence of the opening cost rate for central collection sites: If the opening cost rate takes a value of 1,000, in the $\bar{d}_{\text {high }}$-case only $0.56 \%$ of customers are served by the PS. Lowering the distance limit (and keeping the opening cost rate at 1,000) results in an increase of the corresponding ratio to $16.82 \%$. In contrast, assuming a higher opening cost rate of 10,000 and $\bar{d}_{h i g h}$, only $9.97 \%$ of customers are served by the PS.

Table 12 shows the corresponding average target function values for the three opening cost rates in total, and are broken down by the different cost components of the target function for both waste collection systems. For more detailed results, the reader is referred to Table 25 in the appendix. Table 12 shows that total costs rise when the opening cost rates rise. The decreasing number of central collection sites opened and a share of customers served by the PS cannot compensate for the higher opening cost rates. As mentioned above, if the opening cost rate for central collection sites equals $C^{F}=5$, a pure $\mathrm{BS}$ is used. This is also reflected by the fact that the corresponding routing costs for PS vehicles are zero. Applying the two cost rates of $C^{F}=1,000$ and $C^{F}=10,000$, the higher the opening cost rate, the 
Table 12: Average objective function values and their components for different cost rates

\begin{tabular}{lrrrrrrrr}
\hline & & \multicolumn{4}{c}{ Cost of the BS } & & \multicolumn{2}{c}{ Cost of the PS } \\
\cline { 3 - 5 } \cline { 8 - 9 }$C^{F}$ & Total costs & Routing & Container & Opening site & Assignment & & Routing & Bins \\
\hline 5 & $2,493.52$ & $2,071.30$ & 340.87 & 58.22 & 23.13 & & 0.00 & 0.00 \\
1,000 & $11,228.71$ & $2,052.52$ & 304.98 & $7,477.78$ & 21.78 & & $1,289.60$ & 82.04 \\
10,000 & $74,238.40$ & $2,044.44$ & 289.12 & $69,333.33$ & 20.86 & & $2,402.60$ & 148.04
\end{tabular}

Note. In the component "Assignment" are subsumed the costs incurred for the assignment of customers to central collection sites. This type of cost as well as opening costs are incurred only in the BS and not in the PS. The routing costs correspond to the distances that the assigned vehicles have to travel.

more customers are served by the PS, the higher the bin purchase costs and the further the distances PS vehicles have to travel to collect waste. In turn, the distances covered by the BS vehicles and the costs for purchasing containers for central collection sites decrease.

An even more detailed overview of the waste collection costs is provided in Table 13 The table shows the average costs per customer depending on the total number of customers per instance and both distance limits. In addition to the total costs per customer, also shown are the operational costs incurred by routing the collection vehicles of both types (BS and PS); these costs correspond to the distances driven by the vehicles. The lowest costs per opening cost rate and distance limit are highlighted in grey. For the $\bar{d}_{l o w}$-case, it can be seen that both the total costs and the routing costs per customer contained therein decrease as the number of customers increases.

However, this does not apply to opening cost rates of $C^{F}=1,000$ and $C^{F}=10,000$ under the high distance limit. In the case of an opening cost rate of $C^{F}=10,000$, on average, total cost per customer rises continuously as the number of customers increases. This is due to the fact that the few opened central collection sites are already called on with a very high frequency. For additional customers, new (expensive) central collection sites have to be built because capacity at the sites cannot be increased by raising the frequency (see Table 25 in the appendix). It must also be kept in mind that as the number of customers in a set increases, no additional potential sites are available. The central collection sites that best fit the instance set may have already been established. The routing costs per customer decrease as the number of customers increases (regardless of the distance limit and the opening costs), but cannot compensate the higher opening costs.

It is also worth mentioning that using a high distance limit instead of a low distance limit can con-

Table 13: Average total cost (routing cost) per customer

\begin{tabular}{|c|c|c|c|c|c|c|}
\hline \multirow[b]{2}{*}{$n_{c}$} & \multicolumn{3}{|c|}{$\overline{\bar{d}_{\text {low }}}$} & \multicolumn{3}{|c|}{$\overline{\bar{d}_{h i g h}}$} \\
\hline & $C^{F}=5$ & $C^{F}=1,000$ & $C^{F}=10,000$ & $C^{F}=5$ & $C^{F}=1,000$ & $C^{F}=10,000$ \\
\hline 500 & $4.96(4.25)$ & $19.01(9.76)$ & $97.61(10.34)$ & $1.60 \quad(1.18)$ & $9.15 \quad(1.84)$ & $57.30 \quad(4.79)$ \\
\hline 1,000 & 3.89 (3.48) & $14.42(5.63)$ & $87.74 \quad(5.94)$ & $1.41 \quad(1.03)$ & $8.14 \quad(1.31)$ & $61.26 \quad(2.83)$ \\
\hline 1,500 & $2.91(2.53)$ & $12.32(3.72)$ & $83.04 \quad(4.37)$ & $1.34 \quad(0.94)$ & $8.18 \quad(1.15)$ & 62.95 (2.53) \\
\hline Avg. & .42) & 15.25 & .46 & & 4) & 60.50 \\
\hline$\%$ dev. & - & - & - & $-63 \%(-69 \%)$ & $-44 \%(-77 \%)$ & $-32 \%(-51 \%)$ \\
\hline
\end{tabular}

Note. The \% dev.-row shows the average deviations with respect to the $\bar{d}_{\text {low }}$-case. The costs incurred by routing the collection vehicles are shown in parentheses. 
Table 14: Average deviations of costs with respect to the $\bar{d}_{\text {low }}$-case

\begin{tabular}{ccccccccc}
\hline & \multicolumn{2}{c}{$C^{F}=5$} & & \multicolumn{2}{c}{$C^{F}=1,000$} & & \multicolumn{2}{c}{$C^{F}=10,000$} \\
\cline { 2 - 3 }$n_{c}$ & Total & Routing & & Total & Routing & & Total & Routing \\
\hline 500 & $-68 \%$ & $-72 \%$ & & $-52 \%$ & $-81 \%$ & & $-41 \%$ & $-54 \%$ \\
1,000 & $-64 \%$ & $-70 \%$ & & $-44 \%$ & $-77 \%$ & & $-30 \%$ & $-52 \%$ \\
1,500 & $-54 \%$ & $-57 \%$ & & $-34 \%$ & $-69 \%$ & & $-24 \%$ & $-42 \%$ \\
\hline
\end{tabular}

siderably reduce total costs (from $32 \%$ to $63 \%$ on average). Routing costs can even be reduced by an average of $77 \%$, which is particularly important in times of $\mathrm{CO}_{2}$ minimization. However, the more customers there are in the set, the more the effect of the distance limit decreases, as shown in Table 14 As the number of customers increases, the number of open central collection sites in the set also increases, which reduces the constraints on assigning customers to central collection sites due to the lower distance limit.

\subsubsection{Sensitivity analysis regarding the maximum tour length of PS vehicles.}

So far, the share of customers served by means of the PS has been relatively small. At the same time, the results have shown that the effect of higher opening cost rates for central collection sites on the share of customers served by the PS is comparatively small (compared with the effect of a lower distance limit). The question therefore arises of what limits the share of PS customers. The basic idea of a mixed system was to use the PS to serve customers who are located far away from central collection sites and for whom it would not be cost-effective to set up (expensive) central collection site. Therefore, a sensitivity analysis was performed with respect to the maximum tour length of the PS vehicles. To this end, it was assumed that the maximum tour length is the same for both vehicle types. This may make it difficult to serve widely dispersed customer locations. The calculations performed were repeated (5 runs per setting and instance), this time doubling the maximum tour length for the PS vehicles compared with those of the BS vehicles. For Set a, this results in a maximum tour length of $D_{P S}=1200 ; D_{P S}=2300$ for Set b; and $D_{P S}=1900$ for Set c. The results in Table 15 show the average shares of PS customers per instance, cost rate, and distance level. In fact, the proportion of customers now served by the PS is considerably higher. In some settings, a pure PS is operating. At the same time, it can be seen that the share of customers served by the PS decreases on average as the number of customers in the instance

Table 15: Share of PS customers with double maximum PS tour length (rounded to whole numbers)

\begin{tabular}{|c|c|c|c|c|c|c|c|c|c|c|}
\hline \multirow[b]{2}{*}{$\bar{d}$} & \multirow[b]{2}{*}{$C^{F}$} & \multicolumn{3}{|c|}{ Set a } & \multicolumn{3}{|c|}{ Set $b$} & \multicolumn{3}{|c|}{ Set c } \\
\hline & & I01 & I02 & I03 & I04 & $\mathrm{I} 05$ & I06 & I07 & I08 & I09 \\
\hline & 1,000 & $36 \%$ & $23 \%$ & $18 \%$ & $77 \%$ & $18 \%$ & $17 \%$ & $42 \%$ & $17 \%$ & $20 \%$ \\
\hline$d_{\text {low }}$ & 10,000 & $50 \%$ & $27 \%$ & $20 \%$ & $100 \%$ & $61 \%$ & $46 \%$ & $84 \%$ & $52 \%$ & $34 \%$ \\
\hline & 1,000 & $0 \%$ & $0 \%$ & $8 \%$ & $0 \%$ & $0 \%$ & $0 \%$ & $0 \%$ & $0 \%$ & $1 \%$ \\
\hline$d_{\text {high }}$ & 10,000 & $32 \%$ & $22 \%$ & $18 \%$ & $100 \%$ & $55 \%$ & $38 \%$ & $82 \%$ & $48 \%$ & $34 \%$ \\
\hline
\end{tabular}

Note. Instances 01 (I01), I04 and I07 contain $n_{c}=500$ customers, I02, I05 and I08 contain $n_{c}=1,000$ customers and I03, I06 and I09 contain $n_{c}=1,500$ customers. 
Table 16: Average objective function values and their components for different cost rates with double maximum PS tour length

\begin{tabular}{lrrrrrrrr}
\hline & & \multicolumn{4}{c}{ Cost of the BS } & & \multicolumn{2}{c}{ Cost of the PS } \\
\cline { 3 - 5 }$C^{F}$ & Total & Routing & Container & Opening site & Assignment & & Routing & Bins \\
\hline 1,000 & $10,983.38$ & $1,857.46$ & 288.69 & $6,688.89$ & 21.03 & & $1,986.52$ & 140.78 \\
10,000 & $52,899.77$ & $1,050.74$ & 189.12 & $43,888.89$ & 13.01 & & $6,936.90$ & 471.47 \\
\hline
\end{tabular}

under consideration increases. This can be explained as follows: The more customers there are to be served, the more sites are opened on average, so the more likely it is that one of these central collection sites is readily available to a customer. Assuming opening costs for a central collection site of 5 , even with a double PS tour length, no customers will be served by the PS. Therefore, only the results for $C^{F}=1,000$ and $C=10,000$ are shown in the table.

To conclude, Table 16 shows the average target function values for each cost rate in total and is broken down by the different cost components of the target function for each waste collection system under the new setting. Especially in the $C^{F}=10,000$-case, doubling the maximum PS tour length seems worthwhile: On average, total costs can be reduced by $28.74 \%$ (see Table 16 for comparison). Assuming an opening cost rate of $C^{F}=1,000$, however, the impact on costs is small: Total costs can be reduced by only $2.19 \%$ on average.

\section{Conclusion}

In this article, a new problem has been introduced into the area of waste collection. Instead of a pure bring or pickup system, the possibility of a mixed system has been explored. To solve the problem, an ALNS was developed, which has been proven in tests for both small and large instances to perform well. From a modelling point of view, a new set of subtour eliminiation constraints was proposed to deal with intermediate facilities, where the waste is unloaded.

Waste collection is usually a public service that is managed locally and is therefore also financed from local budgets (Kaza et al. 2018, see Chapter 5). According to Kaza et al. (2018), the share of expenditure on the waste management system of the total municipal budget varies between $4 \%$ in highincome countries and $19 \%$ in low-income countries. If the waste management system is too expensive, funds from other public areas, such as education or housing, will have to compensate. A cost-effective waste system therefore plays an important role "on the overall health of the city" (Kaza et al., 2018 p. 102). The computational results in this article have shown that the cost of waste collection can be reduced considerably if customers are willing to travel a longer distance to dispose of their waste at central collection sites. Cities and municipalities would therefore be advised to convince customers that they should accept walking a bit if this means a reduction in user fees.

The option of a mixed system is particularly interesting for communities that currently operate with a pure pickup system and are considering introducing a bring system. This can be advantageous, especially for reasons of sustainability, as the distances that collection vehicles have to travel are considerably 
reduced. In this context, a mixed system offers the possibility of a gradual introduction of a bring system. The required investment for central collection sites could be spread over a longer period of time. Larger distance limits can help to keep small the number of central collection points needed. It is interesting also to consider a mixed system against the background of urban growth. As the results of the computational study have shown, the share of customers who are served by the pickup system decreases as the total number of customers in the system increases. If, depending on the cost rate for central collection points, the introduction of a pure bring system is not yet worthwhile, the bring system can be supplemented by a pickup system in the transition period. In this context, the maximum tour length restriction of PS vehicles should be chosen carefully because it may have a strong influence on the choice of systems and the resulting total costs.

An aspect of further research should therefore be a deeper analysis from a managerial point of view. Real-world data should be used to analyze for which cities a system change or a mixed system would be economically worthwhile. What are the costs associated with the different waste collection systems and which (possibly distance-related) user fees do customers have to pay for waste collection? 


\section{References}

Akeb, H., Moncef, B., \& Durand, B. (2018). Building a collaborative solution in dense urban city settings to enhance parcel delivery: An effective crowd model in paris. Transportation Research Part E, 119, 223-233.

Angelelli, E., \& Speranza, M. G. (2002a). The application of a vehicle routing model to a wastecollection problem: Two case studies. Journal of the Operational Research Society, 53, 944-952.

Angelelli, E., \& Speranza, M. G. (2002b). The periodic vehicle routing problem with intermediate facilities. European Journal of Operational Research, 137, 233-247.

Azi, N., Gendreau, M., \& Potvin, J.-Y. (2014). An adaptive large neighborhood search for a vehicle routing problem with multiple routes. Computers \& Operations Research, 41, 167-173.

Bruck, K.-J. (2020). Offensive zur Abschaffung der Hausmüllsammlung in rosa Säcken in Hamburg (Attempts to stop the collection of household waste in pink garbage bags in Hamburg). MÜLL und ABFALL, . doi $10.37307 /$ j.1863-9763.2020.06.06

Coene, S., Arnout, A., \& Spieksma, F. C. R. (2010). On a periodic vehicle routing problem. Journal of the Operational Research Society, 61, 1719-1728.

Cuda, R., Guastaroba, G., \& Speranza, M. G. (2015). A survey on two-echelon routing problems. Computers \& Operations Research, 55, 185-199.

Dantzig, G., Fulkerson, R., \& Johnson, S. (1954). Solution of a large-scale traveling-salesman problem. Journal of the Operational Research Society of America, 2, 393-410.

Demir, E., Bektaş, T., \& Laporte, G. (2012). An adaptive large neighborhood search heuristic for the pollution-routing problem. European Journal of Operational Research, 223, 346-359.

Drexl, M., \& Schneider, M. (2015). A survey of variants and extensions of the location-routing problem. European Journal of Operational Research, 241, 283-308.

European Environment Agency (2016). Municipal waste management - country fact sheet germany. URL: https://www.eionet.europa.eu/etcs/etc-wmge/products/country-profiles accessed June $15,2021$.

François, V., Arda, Y., \& Crama, Y. (2019). Adaptive large neighborhood search for multitrip vehicle routing with time windows. Transportation Science, 53, 1706-1730.

Gambella, C., Maggioni, F., \& Vigo, D. (2019). A stochastic programming model for a tactical solid waste management problem. European Journal of Operational Research, 273, 684-694.

Ghiani, G., Laganà, D., Manni, E., Musmanno, R., \& Vigo, D. (2014). Operations research in solid waste management: A survey of strategic and tactical issues. Computers \& Operations Research, 44, 22-32.

Gläser, S., \& Stücken, M. (2021a). Facility location and waste bin allocation and routing problem (FLWBARP). doi:10.17632/p337573636.1 Mendeley Data, V1.

Gläser, S., \& Stücken, M. (2021b). Introduction of an underground waste container system - model and solution approaches. European Journal of Operational Research, 295, 675-689.

Goeke, D., \& Schneider, M. (2015). Routing a mixed fleet of electric and conventional vehicles. European Journal of Operational Research, 245, 81-99.

Hemmelmayr, V., Doerner, K. F., Hartl, R. F., \& Rath, S. (2013). A heuristic solution method for node 
routing based solid waste collection problems. Journal of Heuristics, 19, 129-156.

Hemmelmayr, V., Smilowitz, K., \& de La Torre, L. (2017). A periodic location routing problem for collaborative recycling. IISE Transactions, 49, 414-428.

Hemmelmayr, V. C., Cordeau, J.-F., \& Crainic, T. G. (2012). An adaptive large neighborhood search heuristic for two-echelon vehicle routing problems arising in city logistics. Computers \& Operations Research, 39, 3215-3228.

Hemmelmayr, V. C., Doerner, K. F., Hartl, R. F., \& Vigo, D. (2014). Models and algorithms for the integrated planning of bin allocation and vehicle routing in solid waste management. Transportation Science, 48, 103-120.

Kaza, S., Yao, L., Bhada-Tata, P., \& van Woerden, F. (2018). What a Waste 2. 0: A Global Snapshot of Solid Waste Management To 2050. Urban Development Ser. Washington, D. C: World Bank Publications.

Macrina, G., Di Puglia Pugliese, L., Guerriero, F., \& Laporte, G. (2020). Crowd-shipping with time windows and transshipment nodes. Computers \& Operations Research, 113, 104806.

Mamaghani, E. J., \& Davari, S. (2020). The bi-objective periodic closed loop network design problem. Expert Systems with Applications, 144, 113068.

Nguyen, V.-P., Prins, C., \& Prodhon, C. (2012). Solving the two-echelon location routing problem by a grasp reinforced by a learning process and path relinking. European Journal of Operational Research, $216,113-126$.

OECD (2015). The Metropolitan Century: Understanding Urbanisation and its Consequences. Paris: OECD.

Pires, A., Martinho, G., Rodrigues, S., \& Gomes, M. I. (2019). Sustainable Solid Waste Collection and Management. Cham: Springer.

Pisinger, D., \& Ropke, S. (2010). Large neighborhood search. In Handbook of metaheuristics (pp. 399-419). Boston: Springer.

Prodhon, C. (2008). A metaheuristic for the periodic location-routing problem. In J. Kalcsics, \& S. Nickel (Eds.), Oper. Res. Proc. 2007 Oper. Res. Proc. Berlin, Heidelberg: Springer.

Prodhon, C., \& Prins, C. (2014). A survey of recent research on location-routing problems. European Journal of Operational Research, 238, 1-17.

Ropke, S., \& Pisinger, D. (2006). An adaptive large neighborhood search heuristic for the pickup and delivery problem with time windows. Transportation Science, 40, 455-472.

Salhi, S., \& Rand, G. K. (1989). The effect of ignoring routes when locating depots. European Journal of Operational Research, 39, 150-156.

Schiffer, M., Schneider, M., Walther, G., \& Laporte, G. (2019). Vehicle routing and location routing with intermediate stops: A review. Transportation Science, 53, 319-343.

Schiffer, M., \& Walther, G. (2018). An adaptive large neighborhood search for the location-routing problem with intra-route facilities. Transportation Science, 52, 331-352.

Schneider, M., \& Drexl, M. (2017). A survey of the standard location-routing problem. Annals of Operations Research, 259, 389-414.

Stadtreinigung Hamburg (2019). Baustein für mehr Effizienz: Entsorgung mit Unterflursystemen. URL: https://www.stadtreinigung.hamburg/export/sites/default/wohnungswirtschaft/downloads/ 
broschueren/Broschure-UFS.pdf accessed July 28, 2021.

SULO Deutschland GmbH (2021). Underground systems: Iceberg - the cleanest possible solution. URL: https://de.sulo.com/en/solutions/product-solutions/underground-systems//accessed July 28, 2021.

Zhou, L., Baldacci, R., Vigo, D., \& Wang, X. (2018). A multi-depot two-echelon vehicle routing problem with delivery options arising in the last mile distribution. European Journal of Operational Research, 265, 765-778.

Zhou, L., Lin, Y., Wang, X., \& Zhou, F. (2017). Model and algorithm for bilevel multisized terminal location-routing problem for the last mile delivery. International Transactions in Operational Research, 187, 339. 


\section{A. Appendix}

The WCPSTO can be formulated as follows:

Table 17: Indices used.

\begin{tabular}{ll}
\hline$b$ & Receptacle (container or bin) type index \\
$c$ & Visit combination index \\
$i, j, h, k$ & Indices for sites; where $i=j=h=k=0$ for the depot \\
$l$ & Vehicle index \\
$r$ & Number of visits (frequency) \\
$t$ & Day index \\
\hline
\end{tabular}

Table 18: Sets used.

\begin{tabular}{ll}
\hline$B_{1}$ & Set of container types, which can be served with a vehicle $l \in L_{B S}, B=\left\{1, \ldots, b_{1}\right\}$ \\
$B_{2}$ & Set of bin types, which can be served with a vehicle $l \in L_{P S}, B=\left\{1, \ldots, b_{2}\right\}$ \\
$C_{i}$ & Set of all possible visit combinations for site $i \in N_{P} \cup N_{C}, C_{i}=\{1, \ldots, 12\}$ \\
$L$ & Set of vehicles, $\left\{L_{B S} \cup L_{P S}\right\}$ \\
$L_{B S}$ & Set of vehicles of type $L_{B S}=\left\{1, \ldots, l_{B S}\right\}$ \\
$L_{P S}$ & Set of vehicles of type $L_{P S}=\left\{1, \ldots, l_{P S}\right\}$ \\
$N$ & Set of all nodes, $N=N_{0} \cup N_{C} \cup N_{P} \cup N_{I F}$ \\
$N_{0}$ & Set of depots, $N_{0}=\{0\}$ \\
$N_{C}$ & Set of customers, $N_{C}=\left\{1,2, \ldots, n_{c}\right\}$ \\
$N_{P}$ & Set of potential sites, $N_{P}=\left\{n_{c}+1, \ldots, n_{c}+n\right\}$ \\
$N_{I F}$ & Set of intermediate facilities, $N_{I F}=\left\{n_{C}+n+1, \ldots, n_{c}+n+E\right\}$ \\
$N_{1}$ & Set of nodes consists of $\left\{N_{C} \cup N_{P}\right\}$ \\
$N_{2}$ & Set of nodes consists of $\left\{N_{C} \cup N_{P} \cup N_{I F}\right\}$ \\
$N_{B S}$ & Set of nodes consists of $\left\{N_{P} \cup N_{0} \cup N_{I F}\right\}$ \\
$N_{P S}$ & Set of nodes consists of $\left\{N_{C} \cup N_{0} \cup N_{I F}\right\}$ \\
$N$ & Set of nodes consists of $\left\{N_{C} \cup N_{P} \cup N_{0} \cup N_{I F}\right\}$ \\
$R$ & Set of number of visits, $R=\{1,2,3,6\}$ \\
$T$ & Set of days, $T=\{1, \ldots, 6\}$ \\
\hline
\end{tabular}


Table 19: Parameters used.

\begin{tabular}{ll}
\hline$a_{c t}$ & Indicator whether day $t$ is contained in visit combination $c$ \\
$a_{r}$ & Maximum number of days between two consecutive visits depending on the total \\
& number of visits $r$ in the planning horizon \\
$\alpha_{l}$ & Cost factor for one distance unit traveled with vehicle $l$ \\
$b_{1}$ & Number of container types which can be unloaded by a vehicle $l \in L_{B S}$ \\
$b_{2}$ & Number of bin types which can be unloaded by a vehicle $l \in L_{P S}$ \\
$\beta$ & Cost factor for one distance unit traveled by a customer \\
$C_{b}^{P}$ & Purchase cost for a receptacle of type $b$ \\
$C_{i}^{F}$ & Cost for opening site $i \in N_{P}$ (opening cost rate) \\
$\bar{d}$ & Maximal distance a customer would accept to a central collection site (distance \\
& limit) \\
$d_{i j}$ & Distance/Travel time from site $i$ to $j$ \\
$D$ & Maximum tour length \\
$E$ & Number of intermediate facilities \\
$l_{B S}$ & Number of vehicles of type BS \\
$l_{P S}$ & Number of vehicles of type PS \\
$\lambda_{c r}$ & Indicator stating whether combination $c$ belongs to $r$ \\
$n_{c}$ & Number of customers \\
$n$ & Number of potential central collection sites \\
$Q_{b}$ & Volume capacity of a receptacle of type $b$ \\
$s_{i}$ & Service duration at site $i \in N_{1}$ \\
$u_{b}$ & Space needed by a receptacle of type $b$ \\
$U_{i}$ & Maximum of total available space for receptacles at site $i$ \\
$V_{l}$ & Volume capacity of vehicle $l \in L$ \\
$w$ & Amount of waste produced per day by a customer (waste generation rate) \\
\hline
\end{tabular}


Table 20: Decision variables used.

\begin{tabular}{ll}
\hline$\delta_{i l t}$ & Integer variable \\
$\eta_{i j}$ & Binary variable \\
$f_{i r}$ & Binary variable indicating whether $r$ is used for site $i \in N_{1}(1)$ or not (0) \\
$h_{i}$ & Binary variable indicating whether customer $i \in N_{C}$ is served via PS (1) or not (0) \\
$q_{i}$ & Amount of waste to be collected at site $i \in N_{1}$ at each visit \\
$\theta_{j i}$ & Binary variable indicating whether customer $j \in N_{C}$ is assigned to central collection \\
& site $i \in N_{P}(1)$ or not (0) \\
$\omega_{i h l t}$ & Binary variable \\
$\phi_{i j h l t}$ & Binary variable \\
$\phi_{i j h l t}^{+}$ & Binary variable \\
$\psi_{i h l t}^{+}$ & Integer variable \\
$\psi_{i h l t}^{-}$ & Integer variable \\
$\rho_{i h l t}$ & Binary variable \\
$v_{i j l t}$ & Real variable indicating the load of vehicle $l$ on arc $i j$ on day $t$ \\
$w_{i b}$ & Number of receptacles of type $b$ allocated at site $i$ \\
$x_{i j l t}$ & Binary variable indicating whether vehicle $l$ visits site $j$ immediately after site $i$ on \\
& day $t(1)$ or not $(0)$ \\
$y_{i c}$ & Binary variable, indicating whether visit combination $c \in C_{i}$ is assigned to site $i \in$ \\
& $N_{1}$ \\
$z_{i}$ & Binary variable, indicating whether central collection site $i \in N_{P}$ is opened (1) or \\
$\zeta_{r i}$ & not (0) \\
$\zeta_{r j i}^{+}$ & Binary variable \\
\hline
\end{tabular}




$$
\min \sum_{\substack{i \in N \\ i \neq j}} \sum_{j \in N} \sum_{\substack{l \in L \\ j \neq i}} \sum_{t \in T} \alpha_{l} d_{i j} x_{i j l t}+\sum_{b \in B_{1} \cup B_{2}} \sum_{i \in N_{1}} C_{b}^{P} w_{i b}+\sum_{i \in N_{P}} C^{F} z_{i}+\beta \sum_{i \in N_{C}} \sum_{j \in N_{P}} d_{i j} \theta_{i j}
$$

subject to

$$
\begin{aligned}
& \sum_{i \in N_{P}} \eta_{i j}+h_{j}=1 \\
& \forall j \in N_{C} \\
& \eta_{i j}-\theta_{i j} \leq 0 \\
& \forall i \in N_{P} ; j \in N_{C} \\
& \eta_{i j}-z_{j} \leq 0 \\
& \forall i \in N_{P} ; j \in N_{C} \\
& z_{j}+\theta_{i j}-\eta_{i j} \leq 1 \\
& q_{i}=\sum_{r \in R} a_{r} \cdot w \cdot \zeta_{r i} \\
& \forall i \in N_{P} ; j \in N_{C} \\
& \forall i \in N_{C} \\
& \zeta_{r i} \in\{0,1\} \\
& \forall i \in N_{C} ; r \in R \\
& \forall i \in N_{C} ; r \in R \\
& \forall i \in N_{C} ; r \in R \\
& \zeta_{r i} \leq f_{i r} \\
& \zeta_{r i} \geq h_{i}+f_{i r}-1 \\
& \forall i \in N_{C} ; r \in R \\
& \sum_{j \in N_{P}} d_{i j} \theta_{i j} \leq \bar{d} \\
& \forall i \in N_{C} \\
& q_{i}=\sum_{r \in N_{R}} \sum_{j \in N_{C}} a_{r} \cdot w \cdot \zeta_{r j i}^{+} \\
& \forall i \in N_{P} \\
& \zeta_{r j i}^{+} \leq \theta_{i j} \\
& \forall i \in N_{P} ; j \in N_{C} ; r \in R \\
& \zeta_{r j i}^{+} \leq f_{i r} \\
& \zeta_{r j i}^{+} \geq \theta_{i j}+f_{i r}-1 \\
& \zeta_{r j i}^{+} \in\{0,1\} \\
& \sum_{b \in B_{1}} Q_{b} w_{i b}-q_{i} \geq 0 \\
& \sum_{b \in B_{1}} w_{i b} u_{b} \leq U_{i} \\
& \sum_{b \in B_{2}} Q_{b} w_{i b}-q_{i} \geq 0 \\
& \forall i \in N_{P} ; j \in N_{C} ; r \in R \\
& \forall i \in N_{P} ; j \in N_{C} ; r \in R \\
& \forall i \in N_{P} ; j \in N_{C} ; r \in R \\
& \forall i \in N_{P} \\
& \forall i \in N_{P} \\
& \forall i \in N_{C} \\
& \sum_{b \in B_{2}} w_{i b} u_{b} \leq U_{i} \\
& \forall i \in N_{C} \\
& \sum_{c \in C_{i}} y_{i c}=z_{i} \\
& \forall i \in N_{P} \\
& \sum_{c \in C_{i}} y_{i c}=h_{i} \\
& \forall i \in N_{C} \\
& \sum_{c \in C_{i}} y_{i c} \lambda_{c r}=f_{i r} \\
& \forall i \in N_{1} ; r \in R \\
& \sum_{\substack{j \in N_{B S} \\
j \neq i}} \sum_{l \in L_{1}} x_{i j l t}-\sum_{c \in C_{i}} a_{c t} y_{i c}=0 \\
& \forall i \in N_{D} ; t \in T \\
& \sum_{\substack{j \in N_{P S} \\
j \neq i}} \sum_{l \in L_{2}} x_{i j l t}-\sum_{c \in C_{i}} a_{c t} y_{i c}=0
\end{aligned}
$$




$$
\begin{aligned}
& \sum_{\substack{i \in N \\
i \neq h}} x_{i h l t}-\sum_{\substack{j \in N \\
j \neq h}} x_{h j l t}=0 \\
& \sum_{j \in N_{2}} x_{0 j l t} \leq 1 \\
& x_{i j l t} \leq \sum_{h \in N_{2}} x_{0 h l t} \\
& \sum_{i \in N} \sum_{\substack{j \in N \\
j \neq i}}\left(d_{i j}+s_{i}\right) x_{i j l t} \leq D \\
& x_{i 0 l t}=0 \\
& v_{i j l t}=0 \\
& v_{0 j l t}=0 \\
& x_{i j l t}=0 \\
& \sum_{i \in N} v_{i j l t}+q_{j} \sum_{\substack{i \in N \\
i \neq j}} x_{i j l t}=\sum_{i \in N} v_{j i l t} \\
& v_{i j l t} \leq V_{l} x_{i j l t} \\
& \eta_{i j} \in\{0,1\} \\
& f_{\text {ir }} \in\{0,1\} \\
& h_{i} \in\{0,1\} \\
& q_{i} \geq 0 \\
& \theta_{m i} \in\{0,1\} \\
& v_{i j l t} \geq 0 \\
& w_{i b} \geq 0 \text { and integer } \\
& x_{i j l t} \in\{0,1\} \\
& y_{i c} \in\{0,1\} \\
& z_{i} \in\{0,1\} \\
& \delta_{i l t} \geq 1 \text { and integer } \\
& \delta_{i l t} \leq\left(n_{c}+n\right) \\
& \delta_{i l t}-\delta_{j l t}+1 \leq\left(n_{c}+n\right)\left(1-x_{i j l t}-\sum_{h \in N_{I F}} \phi_{i j h l t}^{+}\right) \\
& \delta_{i l t}-\delta_{j l t}+1 \leq\left(n_{c}+n\right)\left(1-x_{i j l t}-\sum_{h \in N_{I F}}\left(\phi_{i j h l t}^{+}+\psi_{i h l t}^{-}\right)\right) \\
& \phi_{i j h l t}^{+} \in\{0,1\} \\
& \phi_{i j h l t} \in\{0,1\} \\
& \phi_{i j h l t}^{+} \geq x_{i h l t}+x_{h j l t}-1 \\
& \phi_{i j h l t}^{+} \leq\left(1-\phi_{i j h l t}\right) \cdot\left(n_{c}+n\right) \\
& \phi_{i j h l t}^{+} \leq x_{i h l t}+x_{h j l t}-1+\phi_{i j h l t} \cdot\left(n_{c}+n\right)
\end{aligned}
$$$$
\forall l \in L ; t \in T
$$$$
\forall i \in N_{2} ; j \in N ; \quad l \in L ; t \in T
$$$$
\forall l \in L ; t \in T
$$$$
\forall i \in N_{1} ; l \in L ; t \in T
$$$$
\forall i \in N_{I F} ; j \in N ; l \in L ; t \in T
$$$$
\forall j \in N ; l \in L ; t \in T
$$$$
\forall i, j \in N_{I F} ; l \in L ; t \in T
$$$$
\forall j \in N_{1} ; l \in L ; t \in T
$$$$
\forall i, j \in N ; l \in L ; t \in T
$$$$
\forall i \in N_{C} ; j \in N_{P}
$$$$
\forall i \in N_{1} ; r \in R
$$$$
\forall i \in N_{C}
$$$$
\forall i \in N_{I}
$$$$
\forall i \in N_{P} ; m \in N_{C}
$$$$
\forall i, j \in N ; l \in L ; t \in T
$$$$
\forall i \in N_{1} ; b \in B
$$$$
\forall i, j \in N ; i \neq j ; l \in L ; t \in T
$$$$
\forall i \in N_{1} ; c \in C_{i}
$$$$
\forall i \in N_{P}
$$$$
\forall i \in N_{1} ; l \in L ; t \in T
$$$$
\forall i \in N_{1} ; l \in L ; t \in T
$$

$\forall i, j \in N_{I} ; i \neq j ; l \in L ; t \in T$

$\forall i, j \in N_{1} ; i=j ; l \in L ; t \in T$

$$
\begin{aligned}
& \forall i, j \in N_{1} ; l \in L ; t \in T \\
& \forall i, j \in N_{1} ; l \in L ; t \in T
\end{aligned}
$$

$\forall i, j \in N_{1} ; h \in N_{I F} ; l \in L ; t \in T$

$\forall i, j \in N_{1} ; h \in N_{I F} ; l \in L ; t \in T$

$\forall i, j \in N_{1} ; h \in N_{I F} ; l \in L ; t \in T$

$\forall i \in N_{1} ; h \in N_{I F} ; l \in L ; t \in T$ 


$$
\begin{aligned}
& \psi_{i h l t}^{+} \geq 0 \\
& \psi_{i h l t}^{+} \geq x_{i h l t}-2+\sum_{j \in N_{P} \cup N_{0}} x_{h j l t} \\
& \psi_{i h l t}^{+} \leq x_{i h l t}-2+\sum_{j \in N_{P} \cup N_{0}} x_{h j l t}+\left(n_{c}+n\right) \cdot \omega_{i h l t} \\
& \psi_{i h l t}^{+} \geq x_{i h l t}-2+\sum_{j \in N_{C} \cup N_{0}} x_{h j l t} \\
& \psi_{i h l t}^{+} \leq x_{i h l t}-2+\sum_{j \in N_{P} \cup N_{0}} x_{h j l t}+\left(n_{c}+n\right) \cdot \omega_{i h l t} \\
& \psi_{i h l t}^{+} \leq 0+\left(n_{c}+n\right) \cdot\left(1-\omega_{i h l t}\right) \\
& \rho_{\text {ihlt }} \in\{0,1\} \\
& \psi_{\text {ihlt }}^{-} \leq \psi_{\text {ihlt }}^{+} \\
& \psi_{\text {ihlt }}^{-} \leq x_{\text {ihlt }} \\
& \psi_{\text {ihlt }}^{-} \geq x_{\text {ihlt }}-\rho_{\text {ihlgt }} \cdot\left(n_{c}+n\right) \\
& \psi_{\text {ihlt }}^{-} \geq \psi_{\text {ihlt }}^{+}-\left(n_{c}+n\right) \cdot\left(1-\rho_{\text {ihlt }}\right) \\
& x_{\text {ihlt }}-\psi_{\text {ihlt }}^{+} \leq\left(n_{c}+n\right) \cdot \rho_{\text {ihlt }} \\
& \psi_{i h l t}^{+}-x_{i h l t} \leq\left(n_{c}+n\right) \cdot\left(1-\rho_{i h l t}\right)
\end{aligned}
$$

$\forall i \in N_{1} ; h \in N_{I F} ; l \in L ; t \in T$

$\forall i \in N_{P} ; h \in N_{I F} ; l \in L ; t \in T$

$\forall i \in N_{P}, h \in N_{I F} ; l \in L ; t \in T$

$\forall i \in N_{C} ; h \in N_{I F} ; l \in L ; t \in T$

$\forall i \in N_{C}, h \in N_{I F} ; l \in L ; t \in T$

$\forall i \in N_{1} ; h \in N_{I F} ; l \in L ; t \in T$

$\forall i \in N_{1} ; h \in N_{I F} ; l \in L ; t \in T$

$\forall i \in N_{1} ; h \in N_{I F} ; l \in L ; t \in T$

$\forall i \in N_{1} ; h \in N_{I F} ; l \in L ; t \in T$

$\forall i \in N_{1} ; h \in N_{I F} ; l \in L ; t \in T$

$\forall i \in N_{1} ; h \in N_{I F} ; l \in L ; t \in T$

$\forall i \in N_{1} ; h \in N_{I F} ; l \in L ; t \in T$

$\forall i \in N_{1} ; h \in N_{I F} ; l \in L ; t \in T$. 
Table 21: Set of neighborhood structures within the ALNS for the PS in accordance to Hemmelmayr et al. (2014) and the BS in accordance to Gläser \& Stücken (2021a)

\begin{tabular}{|c|c|c|c|}
\hline$\kappa$ & Operator & Min. \# PS customers & Max. \# PS customers \\
\hline 1 & Change combination & 1 & 1 \\
\hline 2 & Change combination & 1 & 2 \\
\hline 3 & Change combination & 1 & 3 \\
\hline$\kappa$ & Operator & Min. segment length & Max. segment length \\
\hline 4 & Intertrip move & 1 & $\min \left(1, n_{c}\right)$ \\
\hline 5 & Intertrip move & 1 & $\min \left(2, n_{c}\right)$ \\
\hline 6 & Intertrip move & 1 & $\min \left(3, n_{c}\right)$ \\
\hline 7 & Intertrip cross-exchange & 1 & $\min \left(1, n_{c}\right)$ \\
\hline 8 & Intertrip cross-exchange & 1 & $\min \left(2, n_{c}\right)$ \\
\hline 9 & Intertrip cross-exchange & 1 & $\min \left(3, n_{c}\right)$ \\
\hline 10 & Intertour move & 1 & $\min \left(1, n_{c}\right)$ \\
\hline 11 & Intertour move & 1 & $\min \left(2, n_{c}\right)$ \\
\hline 12 & Intertour move & 1 & $\min \left(3, n_{c}\right)$ \\
\hline 13 & Intertour cross-exchange & 1 & $\min \left(1, n_{c}\right)$ \\
\hline 14 & Intertour cross-exchange & 1 & $\min \left(2, n_{c}\right)$ \\
\hline 15 & Intertour cross-exchange & 1 & $\min \left(3, n_{c}\right)$ \\
\hline$\kappa$ & Operator & Min. \# PS customers & Max. \# PS customers \\
\hline 16 & Change frequency & 1 & 1 \\
\hline 17 & Change frequency & 1 & 2 \\
\hline 18 & Change frequency & 1 & 3 \\
\hline$\kappa_{U W C}$ & Operator & Min. \# central collection sites & Max. \# central collection sites \\
\hline 19 & Change combination & 1 & 1 \\
\hline 20 & Change combination & 1 & 2 \\
\hline 21 & Change combination & 1 & 3 \\
\hline$\kappa$ & Operator & Min. segment length & Max. segment length \\
\hline 22 & Intertrip move & 1 & $\min (1, \mathrm{n})$ \\
\hline 23 & Intertrip move & 1 & $\min (2, n)$ \\
\hline 24 & Intertrip move & 1 & $\min (3, \mathrm{n})$ \\
\hline 25 & Intertrip cross-exchange & 1 & $\min (1, \mathrm{n})$ \\
\hline 26 & Intertrip cross-exchange & 1 & $\min (2, \mathrm{n})$ \\
\hline 27 & Intertrip cross-exchange & 1 & $\min (3, \mathrm{n})$ \\
\hline 28 & Intertour move & 1 & $\min (1, \mathrm{n})$ \\
\hline 29 & Intertour move & 1 & $\min (2, n)$ \\
\hline 30 & Intertour move & 1 & $\min (3, \mathrm{n})$ \\
\hline 31 & Intertour cross-exchange & 1 & $\min (1, n)$ \\
\hline 32 & Intertour cross-exchange & 1 & $\min (2, n)$ \\
\hline 33 & Intertour cross-exchange & 1 & $\min (3, n)$ \\
\hline$\kappa$ & Operator & Min. \# central collection sites & Max. \# central collection sites \\
\hline 34 & Change frequency & 1 & 1 \\
\hline 35 & Change frequency & 1 & 2 \\
\hline 36 & Change frequency & 1 & 3 \\
\hline$\kappa$ & Operator & \# closings & \# openings \\
\hline 37 & ADD central collection site & 0 & 1 \\
\hline 38 & DROP central collection site & 1 & 0 \\
\hline 39 & SWAP central collection site & 1 & 1 \\
\hline
\end{tabular}


Table 22: Detailed results: ALNS vs. VNS with $\bar{d}_{\text {low }}$ and $C^{F}=5$

Target function values

\begin{tabular}{|c|c|c|c|c|c|c|}
\hline & & \\
\hline & & $\operatorname{ALNS} T_{0}=7$ & ALNS $T_{0}=20$ & VNS & ALNS $T_{0}=7$ & ALNS $T_{0}=20$ \\
\hline \multirow{4}{*}{ I01 } & best & $2,895.08$ & $2,988.51$ & $3,370.27$ & $-14.10 \%$ & $-11.33 \%$ \\
\hline & avg. & $2,967.62$ & $3,034.98$ & $3,541.06$ & $-16.19 \%$ & $-14.29 \%$ \\
\hline & worst & $3,093.93$ & $3,108.24$ & $3,706.42$ & $-16.53 \%$ & $-16.14 \%$ \\
\hline & avg. time & $4,383.41$ & $3,059.28$ & $2,415.12$ & & \\
\hline \multirow{4}{*}{$\mathrm{I} 02$} & best & $4,064.14$ & $4,139.95$ & $5,009.61$ & $-18.87 \%$ & $-17.36 \%$ \\
\hline & avg. & $4,163.87$ & $4,189.08$ & $5,124.00$ & $-18.74 \%$ & $-18.25 \%$ \\
\hline & worst & 4,229.11 & $4,274.30$ & $5,247.33$ & $-19.40 \%$ & $-18.54 \%$ \\
\hline & avg. time & $4,198.44$ & $3,123.91$ & $3,498.88$ & & \\
\hline \multirow{4}{*}{ I03 } & best & $4,503.40$ & $4,661.95$ & $5,724.30$ & $-21.33 \%$ & $-18.56 \%$ \\
\hline & avg. & 4,636.31 & $4,729.26$ & $5,781.08$ & $-19.80 \%$ & $-18.19 \%$ \\
\hline & worst & $4,732.69$ & $4,763.61$ & $5,812.00$ & $-18.57 \%$ & $-18.04 \%$ \\
\hline & avg. time & $4,284.88$ & $3,267.12$ & $2,687.11$ & & \\
\hline \multirow{4}{*}{ I04 } & best & $2,616.91$ & 2,328.07 & $2,459.74$ & $6.39 \%$ & $-5.35 \%$ \\
\hline & avg. & $2,825.86$ & $2,510.83$ & $2,770.17$ & $2.01 \%$ & $-9.36 \%$ \\
\hline & worst & $3,082.41$ & $2,649.51$ & $2,955.10$ & $4.31 \%$ & $-10.34 \%$ \\
\hline & avg. time & $6,554.08$ & $6,031.98$ & $3,466.86$ & & \\
\hline \multirow{4}{*}{ I05 } & best & $4,142.18$ & $4,059.16$ & $4,229.31$ & $-2.06 \%$ & $-4.02 \%$ \\
\hline & avg. & $4,192.58$ & $4,151.38$ & $4,375.27$ & $-4.18 \%$ & $-5.12 \%$ \\
\hline & worst & $4,233.85$ & $4,275.41$ & $4,470.44$ & $-5.29 \%$ & $-4.36 \%$ \\
\hline & avg. time & $5,233.10$ & $4,892.65$ & $7,694.77$ & & \\
\hline \multirow{4}{*}{ I06 } & best & $4,784.11$ & $4,698.20$ & $5,168.12$ & $-7.43 \%$ & $-9.09 \%$ \\
\hline & avg. & $4,851.96$ & $4,744.95$ & $5,357.64$ & $-9.44 \%$ & $-11.44 \%$ \\
\hline & worst & $4,949.04$ & $4,823.12$ & $5,440.64$ & $-9.04 \%$ & $-11.35 \%$ \\
\hline & avg. time & $7,979.63$ & $8,511.73$ & $22,242.78$ & & \\
\hline \multirow{4}{*}{ I07 } & best & $1,897.17$ & $1,807.61$ & $2,094.30$ & $-9.41 \%$ & $-13.69 \%$ \\
\hline & avg. & $2,145.23$ & 1,888.39 & $2,275.57$ & $-5.73 \%$ & $-17.01 \%$ \\
\hline & worst & $2,449.29$ & $2,030.63$ & $2,463.07$ & $-0.56 \%$ & $-17.56 \%$ \\
\hline & avg. time & $3,195.74$ & $3,639.82$ & 2,297.35 & & \\
\hline \multirow{4}{*}{ I08 } & best & $3,019.75$ & $2,821.70$ & $3,286.06$ & $-8.10 \%$ & $-14.13 \%$ \\
\hline & avg. & $3,315.23$ & $2,998.92$ & $3,430.33$ & $-3.36 \%$ & $-12.58 \%$ \\
\hline & worst & $3,509.51$ & $3,098.80$ & $3,581.70$ & $-2.02 \%$ & $-13.48 \%$ \\
\hline & avg. time & $6,565.60$ & $4,337.34$ & $3,217.12$ & & \\
\hline \multirow{4}{*}{ I09 } & best & $3,584.05$ & $3,565.24$ & $4,822.98$ & $-25.69 \%$ & $-26.08 \%$ \\
\hline & avg. & $3,636.49$ & $3,623.73$ & $5,059.40$ & $-28.12 \%$ & $-28.38 \%$ \\
\hline & worst & $3,782.15$ & $\mathbf{3 , 6 5 0 . 3 7}$ & $5,238.69$ & $-27.80 \%$ & $-30.32 \%$ \\
\hline & avg. time & $6,727.20$ & $5,376.10$ & $3,624.46$ & & \\
\hline
\end{tabular}

Note. The lowest values within a set are printed in bold type.
Dev. wrt. to VNS

Co The lowest values within a set are printed in bold type. 
Table 23: Detailed results: ALNS vs. VNS with $\bar{d}_{h i g h}$ and $C^{F}=5$

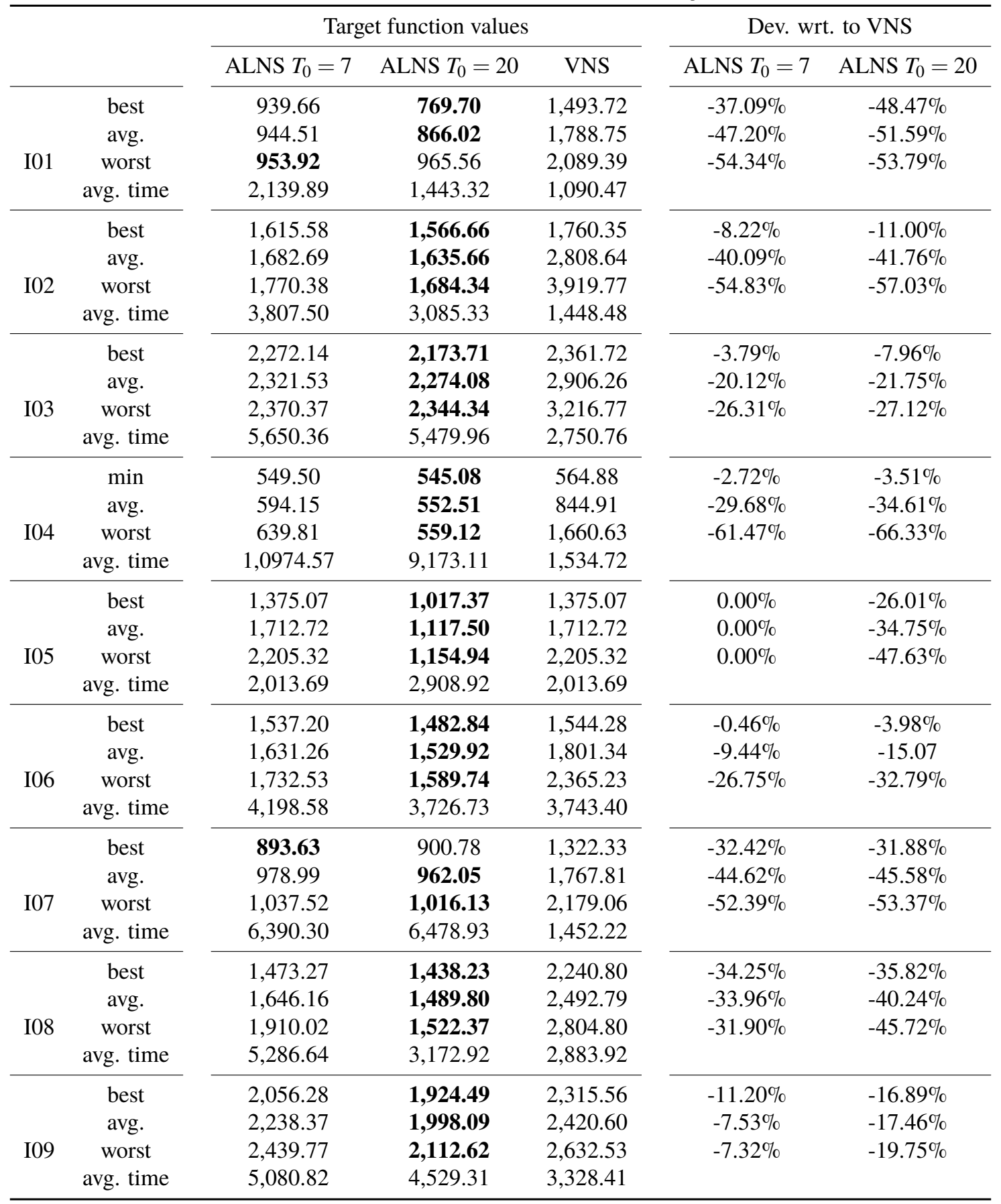

Note. The lowest values within a set are printed in bold type. 
Table 24: Detailed results: ALNS with $C^{F}=1,000$ and $C^{F}=10,000$

\begin{tabular}{|c|c|c|c|c|c|}
\hline & \multicolumn{2}{|c|}{$C^{F}=1,000$} & \multicolumn{2}{|c|}{$C^{F}=10,000$} \\
\hline & & $\bar{d}_{\text {low }}$ & $\bar{d}_{\text {high }}$ & $\bar{d}_{\text {low }}$ & $\bar{d}_{h i g h}$ \\
\hline \multirow{4}{*}{$\mathrm{I} 01$} & best & $9,002.58$ & $4,618.25$ & $53,956.08$ & $32,250.56$ \\
\hline & avg. & $9,517.58$ & $5,128.20$ & $62,325.16$ & $38,165.53$ \\
\hline & worst & $11,399.71$ & $5,925.47$ & $74,790.42$ & $42,212.04$ \\
\hline & avg. time & $6,760.70$ & $3,393.54$ & $10,305.00$ & $3,605.33$ \\
\hline \multirow{4}{*}{$\mathrm{I} 02$} & best & $15,611.50$ & $9,519.85$ & $95,479.96$ & $72,468.98$ \\
\hline & avg. & $17,025.62$ & $10,090.76$ & $109,529.30$ & $80,444.83$ \\
\hline & worst & $18,246.96$ & $10,753.21$ & $125,701.93$ & $91,779.94$ \\
\hline & avg. time & $5,499.49$ & $3,071.29$ & $7,792.48$ & $3,238.58$ \\
\hline \multirow{4}{*}{ I03 } & best & $20,138.32$ & $14,539.35$ & $146,146.85$ & $113,600.38$ \\
\hline & avg. & $21,307.11$ & $14,613.17$ & $161,019.66$ & $117,376.91$ \\
\hline & worst & $22,298.41$ & $14,663.21$ & $197,545.38$ & $122,576.44$ \\
\hline & avg. time & $7,491.58$ & $4,893.62$ & $7,743.21$ & $5,736.03$ \\
\hline \multirow{4}{*}{ I04 } & best & $9,975.27$ & $3,530.06$ & $36,595.48$ & $21,588.18$ \\
\hline & avg. & $10,709.11$ & $3,631.97$ & $44,834.52$ & $22,714.64$ \\
\hline & worst & $11,261.16$ & $3,692.21$ & $56,579.27$ & $23,771.98$ \\
\hline & avg. time & $17,176.60$ & $9,563.48$ & $18,269.07$ & $12,831.24$ \\
\hline \multirow{4}{*}{ I05 } & best & $13,471.36$ & $6,267.92$ & $77,114.17$ & $43,148.29$ \\
\hline & avg. & $14,147.76$ & $6,635.89$ & $83,792.76$ & $47,460.52$ \\
\hline & worst & $14,791.95$ & $7,259.11$ & $88,579.69$ & $53,055.81$ \\
\hline & avg. time & $14,556.20$ & $2,708.48$ & $14,403.82$ & $6,161.40$ \\
\hline \multirow{4}{*}{ I06 } & best & $17,817.50$ & $9,967.21$ & $75,101.84$ & $73,597.27$ \\
\hline & avg. & $18,669.82$ & $10,605.65$ & $109,895.37$ & $76,729.01$ \\
\hline & worst & $20,289.27$ & $11,426.57$ & $138,111.33$ & $85,293.01$ \\
\hline & avg. time & $15,674.79$ & $3,322.83$ & $16,856.68$ & $8,182.64$ \\
\hline \multirow{4}{*}{ I07 } & best & $7,942.50$ & $4,247.30$ & $35,161.79$ & $22,292.83$ \\
\hline & avg. & $8,281.15$ & $4,963.68$ & $39,251.03$ & $25,071.12$ \\
\hline & worst & $8,590.26$ & $5,978.39$ & $45,250.30$ & $32,247.92$ \\
\hline & avg. time & $15,688.88$ & $8,849.29$ & $15,059.20$ & $13,445.63$ \\
\hline \multirow{4}{*}{ I08 } & best & $11,227.71$ & $7,085.14$ & $55,496.43$ & $53,661.57$ \\
\hline & avg. & $11,944.18$ & $7,692.27$ & $69,888.48$ & $55,875.29$ \\
\hline & worst & $12,955.59$ & $8,503.56$ & $76,476.59$ & $62,835.14$ \\
\hline & avg. time & $12,739.11$ & $3,355.18$ & $11,866.67$ & $8,737.81$ \\
\hline \multirow{4}{*}{ I09 } & best & $14,632.39$ & $11,270.58$ & $96,025.51$ & $75,726.47$ \\
\hline & avg. & $15,445.17$ & $11,577.32$ & $102,769.23$ & $89,147.77$ \\
\hline & worst & $16,421.16$ & $12,276.52$ & $107,844.44$ & $104,732.82$ \\
\hline & avg. time & $15,993.10$ & $4,063.38$ & $14,310.16$ & $10,654.20$ \\
\hline
\end{tabular}


Table 25: Detailed results: Average share of PS customers, opened central collection sites and frequencies

\begin{tabular}{|c|c|c|c|c|c|}
\hline $\bar{d}$ & $C^{F}$ & $n_{c}$ & 500 & 1000 & 1500 \\
\hline \multirow{9}{*}{$\bar{d}_{l o w}$} & \multirow{3}{*}{5} & $\%$ PS & $0.00 \%$ & $0.00 \%$ & $0.00 \%$ \\
\hline & & No. sites & 13.33 & 13.73 & 16.87 \\
\hline & & $\varnothing r$ & 3.28 & 5.39 & 5.79 \\
\hline & \multirow{3}{*}{1,000} & $\% \mathrm{PS}$ & $27.35 \%$ & $14.47 \%$ & $8.64 \%$ \\
\hline & & No. sites & 4.33 & 8.33 & 12.27 \\
\hline & & $\varnothing r$ & 5.64 & 5.94 & 5.98 \\
\hline & \multirow{3}{*}{10,000} & $\%$ PS & $29.65 \%$ & $15.79 \%$ & $12.64 \%$ \\
\hline & & No. sites & 4.33 & 8.13 & 11.73 \\
\hline & & $\varnothing r$ & 5.68 & 6.00 & 6.00 \\
\hline \multirow{9}{*}{$\bar{d}_{h i g h}$} & \multirow{3}{*}{5} & $\%$ PS & $0.00 \%$ & $0.00 \%$ & $0.00 \%$ \\
\hline & & No. sites & 5.27 & 8.80 & 11.87 \\
\hline & & $\varnothing r$ & 5.81 & 6.00 & 5.98 \\
\hline & \multirow{3}{*}{1,000} & $\%$ PS & $1.44 \%$ & $0.03 \%$ & $0.20 \%$ \\
\hline & & No. sites & 3.47 & 6.47 & 10.00 \\
\hline & & $\varnothing r$ & 5.96 & 6.00 & 6.00 \\
\hline & \multirow{3}{*}{10,000} & $\% \mathrm{PS}$ & $15.53 \%$ & $7.40 \%$ & $6.99 \%$ \\
\hline & & No. sites & 2.60 & 5.80 & 9.00 \\
\hline & & $\varnothing r$ & 6.00 & 6.00 & 6.00 \\
\hline
\end{tabular}

